\title{
Spectral parameter power series for perturbed Bessel equations
}

\author{
Raúl Castillo-Pérez ${ }^{1}$, Vladislav V. Kravchenko ${ }^{2}$ and Sergii M. Torba ${ }^{2}$ \\ ${ }^{1}$ SEPI, ESIME Zacatenco, Instituto Politécnico Nacional, Av. IPN S/N, C.P. 07738, D.F. Mexico \\ ${ }^{2}$ Departamento de Matemáticas, CINVESTAV del IPN, Unidad Querétaro, Libramiento Norponiente \#2000, \\ Fracc. Real de Juriquilla, Querétaro, Qro. C.P. 76230 MEXICO \\ e-mail: rcastillo@ipn.mx,vkravchenko@math.cinvestav.edu.mx, \\ storba@math.cinvestav.edu.mx
}

October 29, 2018

\begin{abstract}
A spectral parameter power series (SPPS) representation for regular solutions of singular Bessel type Sturm-Liouville equations with complex coefficients is obtained as well as an SPPS representation for the (entire) characteristic function of the corresponding spectral problem on a finite interval. It is proved that the set of zeros of the characteristic function coincides with the set of all eigenvalues of the Sturm-Liouville problem. Based on the SPPS representation a new mapping property of the transmutation operator for the considered perturbed Bessel operator is obtained, and a new numerical method for solving corresponding spectral problems is developed. The range of applicability of the method includes complex coefficients, complex spectrum and equations in which the spectral parameter stands at a first order linear differential operator. On a set of known test problems we show that the developed numerical method based on the SPPS representation is highly competitive in comparison to the best available solvers such as SLEIGN2, MATSLISE and some other codes and give an example of an exactly solvable test problem admitting complex eigenvalues to which the mentioned solvers are not applicable meanwhile the SPPS method delivers excellent numerical results.
\end{abstract}

\section{Introduction}

In the present work the equation

$$
-u^{\prime \prime}+\left(\frac{l(l+1)}{x^{2}}+q(x)\right) u=\lambda\left(r_{1}(x) u^{\prime}+r_{0}(x) u\right), \quad x \in(0, a],
$$

is studied, where $l$ is a real number, $l \geq-\frac{1}{2}, q$ is a complex-valued continuous function on $(0, a]$ satisfying a growth bound $|q(x)| \leq C x^{\alpha}$ at the origin for some $\alpha>-2, r_{0,1} \in C[0, a]$ are complex valued functions, and $\lambda$ is a (complex) spectral parameter. Denote $L=-\frac{d^{2}}{d x^{2}}+\frac{l(l+1)}{x^{2}}+q(x)$. Equations of the form (1.1) appear naturally in many real-world applications after a separation of variables and therefore have received considerable attention (see, e.g., 6], [11, [16, 24], 41]). In preceding publications equation (1.1) was studied under more restrictive conditions, typically for $q$ and $r_{0}$ being real-valued and $r_{1} \equiv 0$. The approach developed in this work does not imply such restrictions and serves both for qualitative study of solutions and spectral problems, as well as for related numerical computation.

The main component in the developed approach is a spectral parameter power series (SPPS) representation for the regular solution of (1.1) obtained under the condition that the auxiliary 
equation $L u_{0}=0$ possesses a regular solution which does not have zeros on $[0, a]$ except at $x=0$. The SPPS representation for solutions of nonsingular linear differential equations and its applications in corresponding scattering and spectral problems were studied in [9], 10], [15], [18], [21], [22], 23], 25], 26], [19], 27], [32], [36] and some other papers. Here, in Section 2 we obtain an analogous result for the perturbed Bessel equation (1.1). The construction and the existence of the required particular solution are addressed in Section [3. For example, when $q(x) \geq 0, x \in(0, a]$ such nonvanishing on $(0, a]$ solution exists. We give an analytic representation for it together with an estimate. Let us emphasize that, in general, $u_{0}$ is allowed to be a complex-valued function, and the existence of such $u_{0}$ for a complex valued $q$ is an open problem.

Under the assumption that $u_{0}$ exists we obtain a dispersion (characteristic) relation for the Sturm-Liouville problem for (1.1) on $[0, a], a<\infty$ (Theorem 4.1). Namely, we construct an entire function $\Phi(\lambda)$ in the form of a Taylor series such that the set of its zeros coincides with the set of all eigenvalues of the Sturm-Liouville problem. This immediately implies the discreteness of the spectrum and offers an efficient method for its computation.

In practical applications of the SPPS method it is often convenient to consider not only series with the centre in the origin $\lambda=0$ but also series with the centre at $\lambda=\lambda_{0}$ where $\lambda_{0}$ is some complex number. In Section 5 we show that this spectral parameter shifting technique is applicable to equation (1.1) and give necessary details.

The SPPS representation allows us to obtain a result on mapping properties of the transmutation operator corresponding to the operator $L$, which was studied, e.g., in [11, 39] and [40]. We show in Section 6 how the transmutation operator acts on certain powers of the independent variable. In the case of nonsingular Schrödinger operators a result of this kind allowed us to advance in the construction of the transmutation operator itself [30] and had applications in constructing complete systems of solutions for some partial differential equations [7, [8], 20].

In Section 7 we explain the numerical implementation of the developed SPPS method for solving Sturm-Liouville problems for (1.1). First, we consider several known test problems, and comparing the obtained results with the results obtained by the best available codes, as SLEIGN2, MATSLISE and some others, we show that our method is highly competitive and gives better or at least comparable results on test problems to which other codes are applicable. Second, we consider an example which involves a different from zero $r_{1}$ in (1.1) and a complex spectrum. Meanwhile SLEIGN2 and MATSLISE are not applicable to problems admitting complex eigenvalues, our method delivers results which are in excellent agreement with the exact data.

\section{Construction of the bounded solution of a perturbed Bessel equation}

Consider a perturbed Bessel operator (also known as a spherical Schrödinger operator)

$$
L=-\frac{d^{2}}{d x^{2}}+\frac{l(l+1)}{x^{2}}+q(x), \quad l \geq-\frac{1}{2}, x \in(0, a]
$$

where the potential $q$ is (in general) a complex-valued continuous function on $(0, a]$ satisfying the growth condition in the origin

$$
q(x)=O\left(x^{\alpha}\right), \quad x \rightarrow 0 \quad \text { for some } \alpha>-2 .
$$

Note that we understand the $O$-notation in the sense of inequality, i.e., there exist a neighborhood $(0, \varepsilon]$ of zero and a constant $C>0$ such that $|q(x)| \leq C x^{\alpha}$ for all $x \in(0, \varepsilon]$. 
If $l \neq 0$ or $q \notin L^{1}(0, a]$, the left endpoint is singular. Despite of that, the equation $L u=0$ possesses a solution $\phi(x)$ which is bounded at $x=0$ and satisfies the following asymptotics at $x=0$

$$
\begin{aligned}
\phi(x) & \sim x^{l+1}, \quad x \rightarrow 0, \\
\phi^{\prime}(x) & \sim(l+1) x^{l}, \quad x \rightarrow 0,
\end{aligned}
$$

see, e.g., [24, Lemma 3.2] for the real-valued potential $q$. We show the explicit construction of the solution with this asymptotics at zero for the general case of complex potentials in Section 3 meanwhile in Section 4 we show that such solution is unique.

Together with $L$ consider a linear differential operator

$$
R u=r_{0} u+r_{1} u^{\prime}
$$

of order at most one, where $r_{0,1} \in C[0, a]$ are complex-valued functions, and consider the following differential equation involving a spectral parameter $\lambda$

$$
L u=\lambda R u \quad \text { or } \quad-u^{\prime \prime}+\left(\frac{l(l+1)}{x^{2}}+q(x)\right) u=\lambda\left(r_{1}(x) u^{\prime}+r_{0}(x) u\right) .
$$

In order to construct a spectral parameter power series representation of a non-singular in zero solution of (2.6) assume that there exists a non-vanishing on $(0, a]$ complex-valued solution $u_{0}$ of the equation

$$
-u_{0}^{\prime \prime}+\left(\frac{l(l+1)}{x^{2}}+q(x)\right) u_{0}=0
$$

satisfying together with its first derivative the asymptotic relations (2.3) and (2.4). Let us define the following system of recursive integrals

$$
\begin{aligned}
\widetilde{X}^{(0)} \equiv 1, \quad \widetilde{X}^{(-1)} \equiv 0, & \begin{cases}\int_{0}^{x} u_{0}(t) R\left[u_{0}(t) \widetilde{X}^{(n-1)}(t)\right] d t, & \text { if } n \text { is odd }, \\
-\int_{0}^{x} \frac{\widetilde{X}^{(n-1)}(t)}{u_{0}^{2}(t)} d t, & \text { if } n \text { is even. }\end{cases}
\end{aligned}
$$

We keep the notation $\widetilde{X}$ for consistency with the notations from other publications on the SPPS method, see, e.g., [27, [19], 29]. Note that for an odd $n$ we have

$$
\begin{aligned}
R\left[u_{0} \widetilde{X}^{(n-1)}\right] & =\left(r_{1} \frac{d}{d x}+r_{0}\right)\left(u_{0} \widetilde{X}^{(n-1)}\right) \\
& =r_{1} u_{0}^{\prime} \widetilde{X}^{(n-1)}-r_{1} u_{0} \frac{\widetilde{X}^{(n-2)}}{u_{0}^{2}}+r_{0} u_{0} \widetilde{X}^{(n-1)}=R\left[u_{0}\right] \widetilde{X}^{(n-1)}-\frac{r_{1}}{u_{0}} \widetilde{X}^{(n-2)} .
\end{aligned}
$$

Hence we can write (2.8) in a different form, which does not require differentiation of the functions $\widetilde{X}^{(n)}$,

$$
\widetilde{X}^{(n)}(x)= \begin{cases}\int_{0}^{x}\left(u_{0}(t) R\left[u_{0}\right](t) \widetilde{X}^{(n-1)}(t)-r_{1}(t) \widetilde{X}^{(n-2)}(t)\right) d t, & \text { if } n \text { is odd, } \\ -\int_{0}^{x} \frac{\widetilde{X}^{(n-1)}(t)}{u_{0}^{2}(t)} d t, & \text { if } n \text { is even. }\end{cases}
$$

The following lemma establishes that all the involved integrals in (2.9) are well defined and provides some estimates for the functions $\widetilde{X}^{(n)}$. 
Lemma 2.1. Let (2.7) admit a solution $u_{0} \in C[0, a] \cap C^{2}(0, a]$ (in general, complex-valued) which does not have other zeros on $[0, a]$ except at $x=0$ and satisfies the asymptotic relations (2.3) and (2.4). Then the system of functions $\left\{\widetilde{X}^{(n)}\right\}_{n=0}^{\infty}$ is well defined by (2.8) or (2.9) and the functions $\widetilde{X}^{(n)}$ satisfy the inequalities

$$
\left|\widetilde{X}^{(2 n)}(x)\right| \leq \frac{C^{2 n} x^{n}}{(2 l+2)_{n}}, \quad\left|\widetilde{X}^{(2 n+1)}(x)\right| \leq \frac{(n+1) C^{2 n+1} x^{2(l+1)+n}}{(2 l+2)_{n+1}},
$$

where $(x)_{n}=\frac{\Gamma(x+n)}{\Gamma(x)}=x(x+1) \cdot \ldots \cdot(x+n-1)$ is the Pochhammer symbol, $C=\max \left\{1, C_{1}, C_{2}, C_{3}\right\}$ and the constants $C_{1}, C_{2}$ and $C_{3}$ are such that for any $t \in(0, a]$ the following inequalities hold

$$
\left|u_{0}(t) R\left[u_{0}\right](t)\right| \leq C_{1} t^{2 l+1}, \quad\left|\frac{1}{u_{0}^{2}(t)}\right| \leq C_{2} t^{-2 l-2}, \quad\left|r_{1}(t)\right| \leq C_{3} .
$$

Remark 2.2. The constants $C_{1}$ and $C_{2}$ in Lemma 2.1 exist due to the fact that $u_{0}$ is a non-vanishing function possessing asymptotics (2.3) and (2.4).

Proof. The proof is by induction. Indeed, for $n=0$ we have $\left|\widetilde{X}^{(0)}(x)\right| \leq 1$ and

$$
\left|\widetilde{X}^{(1)}(x)\right| \leq \int_{0}^{x}\left|u_{0}(t) R\left[u_{0}\right](t)\right| d t \leq \int_{0}^{x} C_{1} t^{2 l+1} d t \leq \frac{C x^{2 l+2}}{2 l+2} .
$$

Assuming that the statement is true for some $n=k$, for $n=k+1$ we obtain

$$
\left|\widetilde{X}^{(2(k+1))}(x)\right| \leq \int_{0}^{x}\left|\frac{\widetilde{X}^{(2 k+1)}(t)}{u_{0}^{2}(t)}\right| d t \leq \int_{0}^{x} \frac{C_{2}}{t^{2 l+2}} \cdot \frac{(k+1) C^{2 k+1} t^{2(l+1)+k}}{(2 l+2)_{k+1}} d t \leq \frac{C^{2 k+2} x^{k+1}}{(2 l+2)_{k+1}}
$$

and

$$
\begin{aligned}
\left|\widetilde{X}^{(2(k+1)+1)}(x)\right| & \leq \int_{0}^{x}\left|u_{0}(t) R\left[u_{0}\right](t) \widetilde{X}^{(2 k+2)}(t)\right| d t+\int_{0}^{x}\left|r_{1}(t) \widetilde{X}^{(2 k+1)}(t)\right| d t \\
& \leq \int_{0}^{x} C_{1} t^{2 l+1} \cdot \frac{C^{2 k+2} t^{k+1}}{(2 l+2)_{k+1}} d t+\int_{0}^{x} C_{3} \cdot \frac{(k+1) C^{2 k+1} t^{2(l+1)+k}}{(2 l+2)_{k+1}} d t \\
& \leq \frac{C^{2 k+3} x^{2 l+2+k+1}}{(2 l+2+k+1) \cdot(2 l+2)_{k+1}}+\frac{C^{2 k+2} \cdot(k+1) x^{2 l+2+k+1}}{(2 l+2+k+1) \cdot(2 l+2)_{k+1}} \\
& \leq \frac{(k+2) C^{2 k+3} x^{2(l+1)+k+1}}{(2 l+2)_{k+2}} .
\end{aligned}
$$

Note that the exponents of the powers of $t$ in all the involved integrands are non-negative, hence all the recursive integrals are well defined.

In the particular case when $r_{1} \equiv 0$, i.e., the right hand side of (2.6) does not depend on the derivative of $u$, the estimates of Lemma 2.1 can be improved, and the following statement is valid.

Lemma 2.3. Under the conditions of Lemma 2.1 assume additionally that $r_{1} \equiv 0$. Then the functions $\widetilde{X}^{(n)}$ defined by (2.8) or (2.9) satisfy the inequalities

$$
\left|\widetilde{X}^{(2 n)}(x)\right| \leq \frac{C^{2 n} x^{2 n}}{2^{2 n} n !(l+3 / 2)_{n}}, \quad\left|\widetilde{X}^{(2 n+1)}(x)\right| \leq \frac{C^{2 n+1} x^{2 n+1+2(l+1)}}{2^{2 n+1} n !(l+3 / 2)_{n+1}},
$$

where $(x)_{n}$ is the Pochhammer symbol and $C=\max \left\{C_{1}, C_{2}\right\}$, where the constants $C_{1}$ and $C_{2}$ are such that for any $t \in(0, a]$ the following inequalities hold

$$
\left|r_{0}(t) u_{0}^{2}(t)\right| \leq C_{1} t^{2 l+2}, \quad\left|\frac{1}{u_{0}^{2}(t)}\right| \leq C_{2} t^{-2 l-2} .
$$


The proof is analogous to that of Lemma 2.1.

The following theorem presents the spectral parameter power series (SPPS) representation of a bounded solution of equation (2.6).

Theorem 2.4. Let (2.7) admit a solution $u_{0} \in C[0, a] \cap C^{2}(0, a]$ (in general, complex-valued) which does not have other zeros on $[0, a]$ except at $x=0$ and satisfies the asymptotic relations (2.3) and (2.4). Then for any $\lambda \in \mathbb{C}$ the function

$$
u=u_{0} \sum_{k=0}^{\infty} \lambda^{k} \widetilde{X}^{(2 k)}
$$

is a solution of (2.6) belonging to $C[0, a] \cap C^{2}(0, a]$ and the series converges uniformly on $[0, a]$. The first derivative of $u$ is given by

$$
u^{\prime}=\frac{u_{0}^{\prime}}{u_{0}} u-\frac{1}{u_{0}} \sum_{k=1}^{\infty} \lambda^{k} \widetilde{X}^{(2 k-1)},
$$

and the series for the first and the second derivatives converge uniformly on an arbitrary compact $K \subset(0, a]$.

Proof. Formally differentiating the series (2.14) twice with the aid of (2.9) we obtain that $u^{\prime}$ should be given by (2.15) and $u^{\prime \prime}$ (after simplification) by $\frac{u_{0}^{\prime \prime}}{u_{0}} u-\lambda r_{0} u-\lambda r_{1} u^{\prime}$. By Lemma2.1 all the involved series converge uniformly on an arbitrary compact $K \subset(0, a]$, hence the formal derivatives coincide with the usual ones. Since by (2.5) and (2.7)

$$
\frac{u_{0}^{\prime \prime}}{u_{0}} u-\lambda r_{0} u-\lambda r_{1} u^{\prime}=\left(\frac{l(l+1)}{x^{2}}+q(x)\right) u-\lambda R[u]
$$

$u$ is indeed a solution of equation (2.6). The relations (2.3) and (2.4) follow from the corresponding asymptotics of $u_{0}$ because by Lemma 2.1 we have $\widetilde{X}^{(2 k)}(x)=o(1)$ and $\frac{1}{u_{0}} \widetilde{X}^{(2 k-1)}=o\left(x^{l}\right)$ for $k \geq 1$.

Remark 2.5. For a regular Sturm-Liouville problem the existence and the construction of the required non-vanishing solution $u_{0}$ present no difficulty since the equation possesses two linearly independent real-valued solutions $u_{1}$ and $u_{2}$ whose zeros alternate and one may choose $u_{0}=u_{1}+i u_{2}$ as such solution. For the singular equation under consideration there is only one solution satisfying the asymptotic conditions (2.3) and (2.4), see Theorem 4.1. Corollary 3.3 establishes that such non-vanishing solution $u_{0}$ exists in the case when $q(x) \geq 0, x \in(0, a]$, and in Remark 5.2 we show that a modified SPPS representation is always possible in the case when $q$ is real valued and bounded from below and $r_{1}$ is real valued.

Remark 2.6. For SPPS representations for solutions of nonsingular Sturm-Liouville equations we refer to [25], 26] and [27. They have been applied in a number of papers to different scattering and spectral problems (see references in the Introduction). For the perturbed Bessel equation, in the case of a real valued potential $q, r_{1} \equiv 0$ and $r_{0} \equiv 1$ an SPPS representation was obtained and used in [24] but without formulas for constructing or estimating the coefficients $\widetilde{X}^{(2 k)}$.

For practical applications the partial sums of the series (2.14) are of the main interest. Based on Lemmas 2.1 and 2.3 the following corollary provides estimates for the difference between the exact solution and the approximate one defined as a partial sum of the series (2.14). The difference is estimated in terms of the remainders of Taylor series of two special functions. 
Corollary 2.7. Under the conditions of Theorem 2.4 consider $u_{N}=u_{0} \sum_{k=0}^{N} \lambda^{k} \widetilde{X}^{(2 k)}$, note that for $N=0$ the right-hand side is equal to $u_{0}$. Then

$$
\left|u(x)-u_{N}(x)\right| \leq \max _{t \in[0, x]}\left|u_{0}(t)\right| \cdot \sum_{k=N+1}^{\infty} \frac{|\lambda|^{k} C^{2 k} x^{k}}{(2 l+2)_{k}} \leq \max _{t \in[0, x]}\left|u_{0}(t)\right| \cdot\left|e^{C^{2}|\lambda| x}-\sum_{k=0}^{N} \frac{\left(C^{2}|\lambda| x\right)^{k}}{k !}\right|,
$$

where the constant $C$ is defined in Lemma 2.1.

Moreover, in the particular case $r_{1} \equiv 0$ the following estimate holds

$$
\begin{aligned}
\left|u(x)-u_{N}(x)\right| & \leq \max _{t \in[0, x]}\left|u_{0}(t)\right| \cdot \sum_{k=N+1}^{\infty} \frac{|\lambda|^{k} C^{2 k} x^{2 k}}{2^{2 k} k !(l+3 / 2)_{k}} \\
& \leq \max _{t \in[0, x]}\left|u_{0}(t)\right| \cdot\left|\frac{2^{l+1 / 2} \Gamma\left(l+\frac{3}{2}\right)}{\left(|\lambda|^{1 / 2} C x\right)^{l+1 / 2}} I_{l+1 / 2}\left(|\lambda|^{1 / 2} C x\right)-\sum_{k=0}^{N} \frac{\left(C^{2}|\lambda| x^{2}\right)^{k}}{2^{2 k} k !(l+3 / 2)_{k}}\right|,
\end{aligned}
$$

where $\Gamma(l+3 / 2)$ is the Gamma function, $I_{l+1 / 2}(x)$ is the modified Bessel function of the first kind and the constant $C$ is defined in Lemma 2.3.

For the proof one should simply compare the majorizing terms corresponding to the even indices in (2.10) and (2.12) with the Taylor expansions for the exponential and the Bessel functions appearing in (2.16) and (2.17).

Example 2.8. Consider the Bessel equation

$$
-\frac{d^{2}}{d x^{2}} u+\frac{l(l+1)}{x^{2}} u=\lambda u
$$

with $l \geq-1 / 2$ and $x \in(0, a]$. The regular solution of this equation satisfying (2.3) and (2.4) is given by the formula

$$
u_{l}(x, \lambda)=\Gamma(l+3 / 2) 2^{l+1 / 2} \lambda^{-\frac{2 l+1}{4}} \cdot \sqrt{x} J_{l+1 / 2}(\sqrt{\lambda} x),
$$

where $J_{l+1 / 2}$ is the Bessel function of the first kind. This solution may be represented as a power series in terms of the parameter $\lambda$,

$$
u_{l}(x, \lambda)=x^{l+1} \sum_{k=0}^{\infty} \frac{(-1)^{k} x^{2 k}}{4^{k} k !(l+3 / 2)_{k}} \lambda^{k},
$$

see [24].

In order to apply Theorem 2.4 consider $u_{0}(x)=x^{l+1}$ as the non-vanishing on $(0, a]$ solution of the equation $-u^{\prime \prime}+\frac{l(l+1)}{x^{2}} u=0$, satisfying (2.3) and (2.4). It is easy to verify that choosing such $u_{0}$ we obtain from (2.9)

$$
\widetilde{X}^{(2 n)}(x)=\frac{(-1)^{n} x^{2 n}}{4^{n} n !(l+3 / 2)_{n}},
$$

i.e., exactly the coefficients from (2.19).

\section{Construction of the particular solution $u_{0}$}

In this section we explain how to construct a particular solution of equation (2.7) satisfying asymptotics (2.3), (2.4) and present some sufficient conditions for this solution to be non-vanishing for $x>0$. 
In order to construct an SPPS representation for the particular solution we rewrite equation (2.7) in the form

$$
y^{\prime \prime}-\frac{l(l+1)}{x^{2}} y=q(x) y .
$$

The equation

$$
y_{0}^{\prime \prime}-\frac{l(l+1)}{x^{2}} y_{0}=0
$$

possesses two solutions

$$
y_{1}(x)=x^{-l} \quad \text { and } \quad y_{2}(x)=x^{l+1} .
$$

For $l \geq-1 / 2$ the second solution is regular and satisfies (2.3) and (2.4). Since the potential $q$ may be singular in the origin, we cannot apply Theorem 2.4 directly. However we may construct the system of recursive integrals in the same way as in (2.9) and only have to show the convergence of the integrals and obtain some estimates justifying the SPPS representation.

Consider the following system of recursive integrals

$$
\begin{aligned}
\tilde{Y}^{(0)} & \equiv 1, \\
\widetilde{Y}^{(n)}(x) & = \begin{cases}\int_{0}^{x} \widetilde{Y}^{(n-1)}(t) t^{2(l+1)} q(t) d t, & \text { for odd } n, \\
\int_{0}^{x} \widetilde{Y}^{(n-1)}(t) t^{-2(l+1)} d t, & \text { for even } n .\end{cases}
\end{aligned}
$$

Note that since the potential $q \in C(0, a]$ satisfies condition (2.2) for some $\alpha>-2$ there exists a constant $C>0$ such that

$$
|q(x)| \leq C x^{\alpha} \quad \text { for all } x \in(0, a] .
$$

Lemma 3.1. Suppose that the complex-valued potential $q \in C(0, a]$ satisfies inequality (3.4) for some $C>0$ and $\alpha>-2$. Then the functions $\widetilde{Y}^{(n)}$ are well defined by (3.3) and the following estimates hold

$$
\begin{aligned}
\left|\widetilde{Y}^{(2 n)}(x)\right| & \leq \frac{C^{n} x^{n(2+\alpha)}}{(2+\alpha)^{2 n} n !\left(\frac{2 l+1}{2+\alpha}+1\right)_{n}}, \\
\left|\widetilde{Y}^{(2 n-1)}(x)\right| & \leq \frac{C^{n} x^{2 l+1+n(2+\alpha)}}{(2+\alpha)^{2 n-1} n !\left(\frac{2 l+1}{2+\alpha}+1\right)_{n}}, \quad x \in(0, a],
\end{aligned}
$$

where $(x)_{n}$ is the Pochhammer symbol.

Proof. The proof can be performed by induction, similarly to the proof of Lemma 2.1, The only difference is that it is possible that an exponent of the power of $t$ under the integral sign be negative, however it is always strictly greater than -1 . Hence all the involved integrals exist.

Proposition 3.2. Suppose that the complex-valued potential $q \in C(0, a]$ satisfies inequality (3.4) for some $C>0$ and $\alpha>-2$. Then the function

$$
u_{0}(x)=x^{l+1} \sum_{k=0}^{\infty} \widetilde{Y}^{(2 k)}(x)
$$

where the functions $\tilde{Y}^{(2 k)}$ are defined by (3.3), is a particular solution of equation (3.1) on $(0, a]$ satisfying asymptotics (2.3), (2.4). Moreover, $u_{0}$ satisfies the following estimate for any $x \in(0, a]$

$$
\left|u_{0}(x)\right| \leq \Gamma\left(\frac{2 l+1}{2+\alpha}+1\right)(2+\alpha)^{\frac{2 l+1}{2+\alpha}} C^{-\frac{2 l+1}{4+2 \alpha}} \sqrt{x} I_{\frac{2 l+1}{2+\alpha}}\left(\frac{2 \sqrt{C x^{2+\alpha}}}{2+\alpha}\right) .
$$


Proof. Due to (3.3) the first and the second derivatives of $u_{0}$ are given by the expressions

$$
u_{0}^{\prime}=(l+1) x^{l} \sum_{k=0}^{\infty} \tilde{Y}^{(2 k)}+x^{-l-1} \sum_{k=1}^{\infty} \widetilde{Y}^{(2 k-1)}
$$

and

$$
\begin{aligned}
u_{0}^{\prime \prime} & =(l+1) l x^{l-1} \sum_{k=0}^{\infty} \tilde{Y}^{(2 k)}+(l+1) x^{-l-2} \sum_{k=1}^{\infty} \tilde{Y}^{(2 k-1)} \\
& -(l+1) x^{-l-2} \sum_{k=1}^{\infty} \tilde{Y}^{(2 k-1)}+x^{l+1} q \sum_{k=1}^{\infty} \tilde{Y}^{(2 k-2)}=\frac{(l+1) l}{x^{2}} u_{0}+q u_{0} .
\end{aligned}
$$

The uniform convergence of all the involved series on an arbitrary compact $K \subset(0, a]$ and hence the possibility of termwise differentiation, follows from Lemma 3.1. The asymptotic relations (2.3) and (2.4) for the function $u_{0}$ follow from the estimates $\widetilde{Y}^{(2 k)}=o(1)$ and $\widetilde{Y}^{(2 k-1)}=o\left(x^{2 l+1}\right), x \rightarrow 0$ for $k \geq 1$, see (3.5) and (3.6). Inequality (3.8) follows from the series representation of the modified Bessel functions of the first kind, see, e.g., [1].

The following corollary provides a sufficient condition for the particular solution constructed in Proposition 3.2 to be non-vanishing.

Corollary 3.3. Under the conditions of Proposition 3.2 assume additionally that $q(x) \geq 0, x \in$ $(0, a]$. Then $u_{0}(x) \geq x^{l+1}$ for any $x \in(0, a]$.

Proof. We obtain from (3.3) and the condition $q(x) \geq 0$ that $\widetilde{Y}^{(n)}(x) \geq 0, n \geq 1$. Hence, $u_{0}(x)=$ $x^{l+1}\left(\widetilde{Y}^{(0)}+\sum_{k=1}^{\infty} \widetilde{Y}^{(2 k)}\right) \geq x^{l+1}$.

\section{Spectral problems}

The classical formulation (see, e.g., 24, 42]) of a spectral problem for a Sturm-Liouville equation of the form $L u=\lambda r u$ with $L$ from (2.1) consists in finding the values of the spectral parameter for which there exists a solution $u(x ; \lambda)$ continuous at $x=0$ and satisfying the following conditions. When $l>1 / 2$,

$$
u(0 ; \lambda)=0
$$

and

$$
\beta u(a ; \lambda)+\gamma u^{\prime}(a ; \lambda)=0
$$

for some $\beta, \gamma \in \mathbb{C}$ such that $|\beta|+|\gamma| \neq 0$.

When $l \in[-1 / 2,1 / 2)$ and since the second linearly independent solution is also square-integrable, an additional boundary condition

$$
\lim _{x \rightarrow 0} x^{l}\left((l+1) u(x ; \lambda)-x u^{\prime}(x ; \lambda)\right)=0,
$$

is imposed (see, e.g., [24]).

Despite the fact that the right-hand side (2.5) of the spectral equation (2.6) may contain the derivative of the unknown function and hence not fit into the basic Sturm-Liouville scheme, we consider the same spectral problem (4.1)-(4.3) for equation (2.6). The following statement gives us a characteristic equation of the spectral problem under the condition that an appropriate nonvanishing solution $u_{0}$ of (2.7) exists. 
Theorem 4.1. Let (2.7) admit a solution $u_{0} \in C[0, a] \cap C^{2}(0, a]$ (in general, complex-valued) which does not have other zeros on $[0, a]$ except at $x=0$ and satisfies the asymptotic relations (2.3) and (2.4). Then the eigenvalues of the problem (2.6), (4.1)-(4.3) coincide with zeros of the entire function

$$
\Phi(\lambda)=\left(\beta u_{0}(a)+\gamma u_{0}^{\prime}(a)\right) \sum_{k=0}^{\infty} \lambda^{k} \widetilde{X}^{(2 k)}(a)-\frac{\gamma}{u_{0}(a)} \sum_{k=1}^{\infty} \lambda^{k} \widetilde{X}^{(2 k-1)}(a),
$$

where the functions $\tilde{X}^{(k)}$ are defined by (2.8) or (2.9).

Proof. Under the condition of the theorem, the function $u$ defined by (2.14) is a solution of (2.6) satisfying the boundary conditions (4.1) and (4.3). The boundary condition (4.2) for the function $u$ coincides with $\Phi(\lambda)=0$, and $\Phi(\lambda)$ is an entire function by Theorem 2.4. It is left to show that there are no more eigenvalues. For that it is sufficient to show that the second linearly independent solution $u_{2}$ of (2.6) does not satisfy either (4.1) or (4.3). We rewrite (2.6) in the form

$$
-u^{\prime \prime}-\lambda r_{1} u^{\prime}+\left(\frac{l(l+1)}{x^{2}}+q-\lambda r_{0}\right) u=0
$$

and introduce the function

$$
p(x)=e^{-\lambda \int_{0}^{x} r_{1}(t) d t} .
$$

Note that

$$
\lim _{x \rightarrow 0} p(x)=1 .
$$

Since the solution $u$ defined by (2.14) satisfies the asymptotic condition (2.3), there exists a constant $b=b(\lambda)>0$ such that $u(x) \neq 0$ for all $x \in(0, b]$. Then the second linearly independent solution is given by the Liouville formula [17, Chap. XI], [37]

$$
u_{2}(x)=-u(x) \int_{x}^{b} \frac{p(t)}{u^{2}(t)} d t, \quad x \in(0, b] .
$$

Assume first that $l>-1 / 2$. It follows from (4.6), asymptotics (2.3), (2.4) and L'Hospital's rule that

$$
u_{2}(x) \sim-\frac{x^{-l}}{2 l+1}, \quad x \rightarrow 0 .
$$

Hence for $l \geq 0$ the second solution $u_{2}$ does not satisfy the boundary condition (4.1). Let now $-1 / 2<l<0$. Recall that by Abel's identity [17. Chap. XI] the Wronskian of $u$ and $u_{2}$ has the form

$$
W=u u_{2}^{\prime}-u^{\prime} u_{2}=p .
$$

Hence using (2.3), (2.4), (4.6) and (4.7) we obtain

$$
u_{2}^{\prime}(x) \sim \frac{l}{2 l+1} x^{-l-1}, \quad x \rightarrow 0
$$

and thus observe that $u_{2}$ cannot satisfy the boundary condition (4.3).

For $l=-1 / 2$ similar reasoning shows that

$$
u_{2}(x) \sim-\sqrt{x} \ln x, \quad x \rightarrow 0 .
$$

We substitute the expression for $u_{2}^{\prime}$ obtained from (4.8) into (4.3) and obtain

$$
x^{-1 / 2}\left(\frac{1}{2} u_{2}-x u_{2}^{\prime}\right)=-\sqrt{x} \frac{p}{u}+\frac{u_{2}}{u} \cdot \frac{u-2 x u^{\prime}}{2 \sqrt{x}} .
$$


For the first term in this expression, from (4.6) and (2.3) we have

$$
\lim _{x \rightarrow 0} \sqrt{x} \frac{p(x)}{u(x)}=1
$$

hence to prove that $u_{2}$ does not satisfy the boundary condition (4.3) it is sufficient to show that the second term is $o(1)$ as $x \rightarrow 0$. Due to the asymptotic relations (2.3) and (4.10) it is sufficient to show that $u-2 x u^{\prime}=O\left(x^{1 / 2+\varepsilon}\right)$ for some $\varepsilon>0$. Taking into account (2.15) we have

$$
u-2 x u^{\prime}=\frac{u}{u_{0}}\left(u_{0}-2 x u_{0}^{\prime}\right)+\frac{2 x}{u_{0}} \sum_{k=1}^{\infty} \lambda^{k} \widetilde{X}^{(2 k-1)} .
$$

As can be seen from (2.10),$\frac{2 x}{u_{0}} \sum_{k=1}^{\infty} \lambda^{k} \widetilde{X}^{(2 k-1)}=o(x)$, hence only the first term is relevant. Since $u \sim u_{0} \sim \sqrt{x}$ as $x \rightarrow 0$, it is sufficient to prove that $u_{0}-2 x u_{0}^{\prime}=O\left(x^{1 / 2+\varepsilon}\right)$. Similarly to (4.10) we obtain that the general solution of equation (2.7) can be represented as $c_{1} \tilde{u}_{1}+c_{2} \tilde{u}_{2}$, where $\tilde{u}_{1}$ is given by (3.7) and satisfies the asymptotic condition (2.3) and $\tilde{u}_{2}$ satisfies the asymptotic condition $\tilde{u}_{2} \sim \sqrt{x} \ln x, x \rightarrow 0$. Since $u_{0} \sim \sqrt{x}$ by the statement of the theorem, it is necessarily of the form (3.7). Now using (3.7), (3.9) and (3.6) we obtain

$$
u_{0}-2 x u_{0}^{\prime}=\sqrt{x} \sum_{k=0}^{\infty} \tilde{Y}^{(2 k)}-\frac{2 x}{2 \sqrt{x}} \sum_{k=0}^{\infty} \tilde{Y}^{(2 k)}-2 \sqrt{x} \sum_{k=1}^{\infty} \tilde{Y}^{(2 k-1)}=O\left(x^{1 / 2+2+\alpha}\right),
$$

where $\alpha$ participates in (2.2), and this finishes the proof for $l=-1 / 2$.

\section{Spectral shift technique}

The SPPS representation given in Theorem 2.4 is based on a particular solution of equation (2.6) for $\lambda=0$. In [27] it was mentioned that for a classic Sturm-Liouville equation it is also possible to construct the SPPS representation of a general solution starting from a non-vanishing particular solution for some $\lambda=\lambda_{0}$. Such procedure is called spectral shift and has already proven its usefulness for numerical applications [27], [18].

We show that a spectral shift technique may be applied to equation (2.6). Let $\lambda_{0}$ be a fixed complex number. We rewrite (2.6) in the form

$$
-u^{\prime \prime}-\lambda_{0} r_{1} u^{\prime}+\left(\frac{l(l+1)}{x^{2}}+q-\lambda_{0} r_{0}\right) u=\tilde{\lambda}\left(r_{1} u^{\prime}+r_{0} u\right),
$$

where $\tilde{\lambda}:=\lambda-\lambda_{0}$. Suppose that $u_{0}$ is a solution of the equation

$$
L_{0} u:=-u^{\prime \prime}-\lambda_{0} r_{1} u^{\prime}+\left(\frac{l(l+1)}{x^{2}}+q-\lambda_{0} r_{0}\right) u=0
$$

such that $u_{0}$ does not vanish on $(0, a]$. Note that $u_{0}$ is a particular solution of (2.6) for $\lambda=\lambda_{0}$. Then the operator $L_{0}$ admits the following Pólya factorization [35]

$$
L_{0} u=-\frac{1}{p u_{0}} \frac{d}{d x} p u_{0}^{2} \frac{d}{d x} \frac{u}{u_{0}}
$$

where

$$
p(x)=e^{\lambda_{0} \int_{0}^{x} r_{1}(s) d s} .
$$


Based on the factorization (5.3) we introduce the following system of recursive integrals

$$
\begin{aligned}
\widetilde{Z}^{(0)} \equiv 1, \quad \widetilde{Z}^{(-1)} \equiv 0, \\
\widetilde{Z}^{(n)}(x)= \begin{cases}\int_{0}^{x} p(t) u_{0}(t) R\left[u_{0}(t) \widetilde{Z}^{(n-1)}(t)\right] d t, & \text { if } n \text { is odd, } \\
-\int_{0}^{x} \frac{\widetilde{Z}^{(n-1)}(t)}{p(t) u_{0}^{2}(t)} d t, & \text { if } n \text { is even. }\end{cases}
\end{aligned}
$$

Similarly to (2.9) the recurrent relation (5.5) can be rewritten as

$$
\widetilde{Z}^{(n)}(x)= \begin{cases}\int_{0}^{x}\left(p(t) u_{0}(t) R\left[u_{0}\right](t) \widetilde{Z}^{(n-1)}(t)-r_{1}(t) \widetilde{Z}^{(n-2)}(t)\right) d t, & \text { if } n \text { is odd, } \\ -\int_{0}^{x} \frac{\widetilde{Z}^{(n-1)}(t)}{p(t) u_{0}^{2}(t)} d t, & \text { if } n \text { is even. }\end{cases}
$$

Theorem 5.1. Let (5.2) admit a solution $u_{0} \in C[0, a] \cap C^{2}(0, a]$ (in general, complex-valued) which does not have other zeros on $[0, a]$ except at $x=0$ and satisfies the asymptotic relations (2.3) and (2.4). Then for any $\lambda \in \mathbb{C}$ the function

$$
u=u_{0} \sum_{k=0}^{\infty}\left(\lambda-\lambda_{0}\right)^{k} \widetilde{Z}^{(2 k)}
$$

is a solution of (2.6) belonging to $C[0, a] \cap C^{2}(0, a]$, and the series (5.7) converges uniformly on $[0, a]$. The series for the first and the second derivatives converge uniformly on an arbitrary compact $K \subset(0, a]$ and the first derivative has the form

$$
u^{\prime}=\frac{u_{0}^{\prime}}{u_{0}} u-\frac{1}{p u_{0}} \sum_{k=1}^{\infty}\left(\lambda-\lambda_{0}\right)^{k} \widetilde{Z}^{(2 k-1)}=u_{0}^{\prime}+\sum_{k=1}^{\infty}\left(\lambda-\lambda_{0}\right)^{k}\left(\widetilde{Z}^{(2 k)}-\frac{\widetilde{Z}^{(2 k-1)}}{p u_{0}}\right) .
$$

Proof. Note that the function $p$ given by (5.4) is continuous and non-vanishing on $[0, a]$. Similarly to the proof of Lemma 2.1 we see that the functions $\widetilde{Z}^{(n)}$ satisfy estimates (2.10) with the constant

$$
C=\max \left\{1, C_{1}, C_{2}, C_{3}\right\},
$$

where

$$
C_{1}=\sup _{t \in(0, a]} \frac{\left|p(t) u_{0}(t) R\left[u_{0}\right](t)\right|}{t^{2 l+1}}, \quad C_{2}=\max _{t \in[0, a]} \frac{t^{2 l+2}}{\left|p(t) u_{0}^{2}(t)\right|}, \quad C_{3}=\max _{t \in[0, a]}\left|r_{1}(t)\right| .
$$

Formal application of the operator $L_{0}$ to (5.7) with the use of Pólya factorization (5.3) and formula (5.5) shows that the function $u$ is a solution of equation (5.1) and hence of (2.6). Estimates for $\widetilde{Z}^{(n)}$ justify the possibility of differentiation of the involved series and show that the function $u$ satisfies (2.3) and (2.4).

Remark 5.2. Suppose that the functions $q$ and $r_{1}$ are real-valued and that the potential $q$ is bounded from below, i.e., there exists a constant $q_{0} \in \mathbb{R}$ such that

$$
q(x) \geq q_{0} \quad \text { for all } x \in(0, a] .
$$

Consider equation (5.2) for $\lambda_{0}=q_{0}$. The particular solution of this equation can be constructed similarly to Section 3 using the generalization of formulas (3.3) according to the factorization (5.3), cf., (2.8) and (5.5). Since the functions $p$ and $q-q_{0}$ are non-negative, similarly to Corollary 3.3 we deduce that the particular solution $u_{0}$ in such case does not have other zeros on $[0, a]$ except at $x=0$. Hence it is possible to construct the SPPS representation of the bounded solution for any equation (2.6) having a real-valued $r_{1}$ and a real-valued bounded from below $q$. 
Remark 5.3. In the case when $r_{1} \equiv 0$, we do not need to introduce the new system of functions $\widetilde{Z}^{(n)}$. The system of functions $\widetilde{X}^{(n)}$ given by (2.9) may be used directly in representation (5.7).

Remark 5.4. For the difference $\max _{x \in[0, a]}\left|u(x)-u_{N}(x)\right|$, where $u_{N}=u_{0} \sum_{k=0}^{N} \lambda^{k} \widetilde{Z}^{(2 k)}$ the estimate (2.16) holds, where $C$ is given by (5.9).

\section{Transmutation operators for perturbed Bessel operators}

We recall a general definition of a transmutation operator from [29] which is a modification of the definition given by Levitan [34]. Let $E$ be a linear topological space and $E_{1}$ its linear subspace (not necessarily closed). Let $A$ and $B$ be linear operators: $E_{1} \rightarrow E$.

Definition 6.1. A linear invertible operator $T$ defined on the whole $E$ such that $E_{1}$ is invariant under the action of $T$ is called a transmutation operator for the pair of operators $A$ and $B$ if it fulfills the following two conditions.

1. Both the operator $T$ and its inverse $T^{-1}$ are continuous in $E$;

2. The following operator equality is valid

$$
A T=T B
$$

or which is the same

$$
A=T B T^{-1} .
$$

Very often in literature the transmutation operators (the term coined by Delsarte and Lions [14]) are called transformation operators.

In [8] for the case of the transmutation operator $T$ corresponding to the pair of operators $A=-\frac{d^{2}}{d x^{2}}+q(x)$ and $B=-\frac{d^{2}}{d x^{2}}$ a mapping property of $T$ was found. It establishes what is the result of action of $T$ on the powers of the independent variable. This mapping property already found many applications in the proofs of completeness of infinite systems of solutions of some linear partial differential equations and in solving initial and spectral problems, see [7], [8], [20], [28], [30], 31]. We present an analogue of the aforementioned mapping property for the transmutation operator for the pair of operators $A=-\frac{d^{2}}{d x^{2}}+q(x)+\frac{l(l+1)}{x^{2}}$ and $B=-\frac{d^{2}}{d x^{2}}+\frac{l(l+1)}{x^{2}}$, constructed in [39], [40].

We recall some results from [39], [40]. Under certain additional conditions on the potential $q$, discussed below, a bounded solution of the equation

$$
-y^{\prime \prime}+\left(q(x)+\frac{l(l+1)}{x^{2}}\right) y=\lambda y
$$

can be represented in the form

$$
y(x, \lambda)=j_{l+1 / 2}(x, \lambda)+\int_{0}^{x} K(x, t) j_{l+1 / 2}(t, \lambda) d t,
$$

where $j_{l+1 / 2}(x, \lambda)=\sqrt{x \sqrt{\lambda}} J_{l+1 / 2}(x \sqrt{\lambda})$ is a solution of the equation

$$
-y^{\prime \prime}+\frac{l(l+1)}{x^{2}} y=\lambda y
$$


and $J_{l+1 / 2}$ is the Bessel function of the first kind. The integral kernel $K$ is the solution of the partial differential equation

$$
\frac{\partial^{2} K(x, t)}{\partial x^{2}}-\frac{l(l+1)}{x^{2}} K(x, t)-q(x) K(x, t)=\frac{\partial^{2} K(x, t)}{\partial t^{2}}-\frac{l(l+1)}{t^{2}} K(x, t)
$$

satisfying the boundary conditions

$$
\frac{d K(x, x)}{d x}=\frac{1}{2} q(x) \quad \text { and } \quad \lim _{t \rightarrow 0} K(x, t) \cdot t^{l}=0 .
$$

Moreover, the integral kernel $K$ satisfies

$$
\sup _{0 \leq x \leq a} \int_{0}^{x}|K(x, t)|^{2} d t<\infty
$$

We denote the operator defined by (6.2) as $\mathbf{T}$. The existence of such operator was established in [40] for the case when $q$ is a continuous function on [0,a] and in [39] for the case when $l$ is an integer and $q$ is a real-valued function satisfying the condition $\int_{0}^{a} t^{m}|q(t)| d t<\infty$ for some $0<m<1 / 2$. It is mentioned in [38] that the results of [2] allow one to extend the existence of the operator $\mathbf{T}$ onto arbitrary real values of the parameter $l$.

The solution $y(x, \lambda)$ in (6.2) differs from the solution $u(x, \lambda)$ satisfying the asymptotic condition (2.3) by the factor $\frac{\lambda^{(l+1) / 2}}{2^{l+1 / 2} \Gamma(l+3 / 2)}$, see [39]. The series expansion of the function $j_{l+1 / 2}(x, \lambda)$ is

$$
j_{l+1 / 2}(x, \lambda)=\sum_{k=0}^{\infty} c_{k} x^{2 k+l+1}, \quad \text { where } c_{k}=c_{k}(\lambda)=\frac{(-1)^{k} \lambda^{k+(l+1) / 2}}{\Gamma(k+1) \Gamma(k+l+3 / 2) 2^{2 k+l+1 / 2}} .
$$

Similarly to [8, Theorem 7] we substitute the functions $y(x, \lambda)$ and $j_{l+1 / 2}(x, \lambda)$ in (6.2) by their series expansions (2.14) and (6.5) and obtain

$$
\begin{aligned}
\frac{\lambda^{(l+1) / 2}}{2^{l+1 / 2} \Gamma\left(l+\frac{3}{2}\right)} u_{0}(x) \sum_{k=0}^{\infty} \lambda^{k} \tilde{X}^{(2 k)}(x) & =\sum_{k=0}^{\infty} c_{k} x^{2 k+l+1}+\int_{0}^{x}\left(K(x, t) \sum_{k=0}^{\infty} c_{k} t^{2 k+l+1}\right) d t \\
& =\sum_{k=0}^{\infty} c_{k}\left(x^{2 k+l+1}+\int_{0}^{x} K(x, t) t^{2 k+l+1} d t\right) \\
& =\sum_{k=0}^{\infty} \frac{(-1)^{k} \lambda^{k+(l+1) / 2}}{\Gamma(k+1) \Gamma\left(k+l+\frac{3}{2}\right) 2^{2 k+l+1 / 2}} \mathbf{T}\left[x^{2 k+l+1}\right] .
\end{aligned}
$$

The function $K(x, \cdot)$ is square-integrable on $[0, x]$, see (6.4), and the function $j_{l+1 / 2}(\cdot, \lambda)$ is the limit of the uniformly convergent series (6.5), hence the possibility to change the order of summation and integration in (6.6) follows from the continuity of the scalar product in $L^{2}[0, a]$.

Since the equality in (6.6) holds for all $x$ and $\lambda$, we finally obtain that

$$
\mathbf{T}\left[x^{2 k+l+1}\right]=(-1)^{k} 2^{2 k} k !\left(l+\frac{3}{2}\right)_{k} u_{0}(x) \widetilde{X}^{(2 k)}(x) .
$$

\section{Numerical implementation and examples}

Based on the results of the previous sections we can formulate a numerical method for solving spectral problems for perturbed Bessel equations. 
1. Find a particular solution $u_{0}$ of equation (2.7) satisfying the asymptotic conditions (2.3) and (2.4). Note that due to the proof of Theorem 4.1 it is sufficient to check that the solution satisfies only the asymptotic condition (2.3). If an analytic expression for the particular solution is unknown, one can use a numerical approximation suggested by Proposition 3.2.

2. Check that the particular solution $u_{0}$ obtained in step 1 is non-vanishing for $x \in(0, a]$. Under the conditions of Corollary 3.3 it is always the case. If the particular solution has zeros on $(0, a]$, the spectral shift technique described in Section 5 can help, either directly as described in Remark 5.2 or by finding a suitable value of $\lambda_{0}$, complex in general.

3. Use partial sums of the series (2.14) and (2.15) (or, correspondingly, (5.7) and (5.8)) to obtain a polynomial

$$
\Phi_{N}(\lambda)=\left(\beta u_{0}(a)+\gamma u_{0}^{\prime}(a)\right)+\sum_{k=1}^{N} \lambda^{k}\left(\left(\beta u_{0}(a)+\gamma u_{0}^{\prime}(a)\right) \tilde{X}^{(2 k)}(a)-\frac{\gamma}{u_{0}(a)} \tilde{X}^{(2 k-1)}(a)\right)
$$

approximating the characteristic function (4.4).

4. Find zeros of the calculated polynomial $\Phi_{N}(\lambda)$.

5. To improve the accuracy of the eigenvalues located farther from the point $\lambda_{0}$, the spectral parameter corresponding to the current particular solution, perform one or several steps of the spectral shift technique.

Since the characteristic function $\Phi(\lambda)$ is analytic, the Rouché theorem from complex analysis, see, e.g., [12], provides the stability of the numerical procedure. Indeed, let $\Gamma$ be an arbitrary simple closed contour on which $\Phi$ does not vanish. Then if the absolute error of approximation $\left|\Phi_{N}-\Phi\right|$ is less than min $\left|\Phi_{N}\right|$ on $\Gamma$, due to Rouché's theorem the functions $\Phi_{N}$ and $\left(\Phi-\Phi_{N}\right)+\Phi_{N}=\Phi$ possess the same number of zeros inside $\Gamma$. Hence the procedure described above does not produce additional (or on the contrary less) zeros whenever $\Phi_{N}$ approximates well enough the function $\Phi$. We illustrate this point below, in Example 7.7.

Before considering numerical examples let us explain how the numerical implementation of the SPPS method was done in this work. All the calculations were performed with the help of Matlab 2010 in the double precision machine arithmetics. The formal powers $\widetilde{X}^{(n)}$ were calculated using the Newton-Cottes 6 point integration formula of 7-th order, see, e.g., [13], modified to perform indefinite integration. We choose $M$ equally spaced points covering the segment of interest and apply the integration formula to overlapping groups of six points. It is worth mentioning that for large values of the parameter $l$ a special care should be taken near the point 0 , because even small errors in the values of $\widetilde{X}^{(2 n+1)}$ after the division by $u_{0}^{2} \sim x^{2(l+1)}$ lead to large errors in the computation of $\widetilde{X}^{(2 n)}$ on the whole interval $[0, a]$. To overcome this difficulty we change the values of $\widetilde{X}^{(2 n+1)}$ in several points near zero to their asymptotic values. This strategy leads to a good accuracy. The computation of the first 100-200 formal powers proved to be a completely feasible task, and even for $M$ being as large as several millions the computation time of the whole set of formal powers is within seconds. In the presented numerical results we specify two parameters: $N$ is the degree of the polynomial $\Phi_{N}$ in (7.1), i.e., the number of the calculated formal powers is $2 N$, and $M$ is the number of points taken on the considered segment for the calculation of integrals.

Let us stress that the formal powers do not depend on the spectral parameter and once calculated can be used for computing the solution and/or the characteristic function $\Phi(\lambda)$ for thousands of different values of the spectral parameter $\lambda$ without any additional significant computation cost. 


\begin{tabular}{cccc}
\hline$n$ & $\lambda_{n}$ (SPPS) & $\lambda_{n}$ (SLEIGN2, [4]) & \multicolumn{1}{c}{$\lambda_{n}$ (Exact) } \\
\hline 1 & 12.1871394680951 & 12.187139459 & 12.1871394680951 \\
2 & 44.257559403500 & 44.257558912 & 44.257559403502 \\
3 & 96.07160483898 & 96.071604502 & 96.07160483884 \\
4 & 167.62571241787 & 167.625711908 & 167.62571242058 \\
5 & 258.91930037169 & 258.919292439 & 258.91930035744 \\
6 & 369.95220905860 & 369.952209262 & 369.95220926235 \\
7 & 500.72440133519 & 500.724370471 & 500.72438147579 \\
8 & 651.23517308180 & 651.235865279 & 651.23579210254 \\
9 & 821.50506498326 & 821.486428982 & 821.48642898238 \\
10 & 988.97560762340 & 1011.476285608 & 1011.47628560802 \\
\hline
\end{tabular}

Table 1: The eigenvalues from Example 7.1 for $c=\frac{5}{16}$, calculated with $N=40$ and $M=5 \cdot 10^{4}$.

There exist several computer codes for the solution of singular Sturm-Liouville problems. We compare our results with the results produced by SLEIGN2 [5] and MATSLISE [33]. Both packages can reliably solve a variety of spectral problems for regular and singular Sturm-Liouville problems and are considered as basic comparison tools, the second package to our best knowledge is one of the most accurate. Both packages work with double precision machine arithmetics and were used with the parameters for the highest possible accuracy goals. It should be mentioned that the applicability of both packages is restricted to the self-adjoint situation and they do not permit neither complex coefficients nor a first order differential operator at a spectral parameter. Thus, to illustrate the performance of the proposed method in a situation when a comparison to the existing software is impossible, in Example 7.7 we consider a problem for which an exact characteristic equation can be written down and compare the obtained numerical results to those calculated from the exact equation.

\subsection{Real spectrum}

Example 7.1. Our first numerical example is the Bessel equation, which we already touched on in Example 2.8. Consider the following spectral problem [4, Example 2]

$$
\left\{\begin{array}{l}
-y^{\prime \prime}+\frac{c}{x^{2}} y=\lambda y, \quad 0<x \leq 1 \\
y(1, \lambda)=0
\end{array}\right.
$$

In this and in all other considered examples the solution $y(x, \lambda)$ should also satisfy the boundary conditions (4.1) and (4.3) at 0 . As follows from (2.18), the eigenvalues of the problem (7.2) coincide with zeros of the Bessel function $J_{\nu}(s)$, where $s=\sqrt{\lambda}$ and $\nu=\sqrt{c+\frac{1}{4}}$.

We compare the results delivered by the SPPS method to those from [4, Example 2] for a particular case $c=\frac{5}{16}$. For the SPPS representation (2.14) the exact particular solution $u_{0}=x^{5 / 4}$ was used. The results are presented in Table 1 together with the exact eigenvalues calculated as squares of zeros of $J_{3 / 4}(s)$ and computed using the Matlab routine besselzero.m by Greg von Winckel.

As can be seen from Table 1, the accuracy of the higher eigenvalues calculated by the SPPS method decreases. To improve the accuracy the spectral shift technique described in Section 5 is applied. We choose that the values of the parameter $\lambda_{0}$ change along a line in the complex plane and are given by $\lambda_{0}^{(n)}=50 n+2 n i, n=1 \ldots 4000$. On each step we compute the solution in the next point $\lambda_{0}^{(n+1)}$ and use this solution as a particular solution for the next step. Since the spectral 


\begin{tabular}{ccccr}
\hline$n$ & $\lambda_{n}$ (exact/MATSLISE) & $\lambda_{n}$ (SPPS) & \multicolumn{1}{c}{$\lambda_{0}$ used } & \multicolumn{1}{c}{$\lambda_{n}$ (SLEIGN2) } \\
\hline 1 & 12.1871394680951 & 12.1871394680951 & $1+0.5 i$ & 12.187139459 \\
2 & 44.257559403502 & 44.257559403500 & $50+2 i$ & 44.257558912 \\
3 & 96.071604838843 & 96.071604838834 & $100+4 i$ & 96.071604502 \\
4 & 167.62571242058 & 167.62571242056 & $150+6 i$ & 167.625711908 \\
5 & 258.91930035744 & 258.91930035742 & $250+10 i$ & 258.919292439 \\
6 & 369.95220926235 & 369.95220926232 & $350+14 i$ & 369.952209262 \\
7 & 500.72438147579 & 500.72438147575 & $500+20 i$ & 500.724370471 \\
8 & 651.23579210254 & 651.23579210250 & $650+26 i$ & 651.235865279 \\
9 & 821.48642898238 & 821.48642898235 & $800+32 i$ & 821.486428982 \\
10 & 1011.47628560802 & 1011.47628560801 & $1000+40 i$ & 1011.476285608 \\
30 & 8956.5077203636 & 8956.5077203638 & $8950+358 i$ & 8956.507721371 \\
50 & 24797.222775294 & 24797.222775296 & $24800+992 i$ & 24796.866878938 \\
75 & 55701.421553437 & 55701.421553167 & $50000+2000 i$ & 55701.328815911 \\
100 & 98942.625835 & 98942.625812 & $100000+4000 i$ & 98943.253862034 \\
\hline
\end{tabular}

Table 2: The eigenvalues from Example 7.1 for $c=\frac{5}{16}$, calculated with the use of the spectral shift technique with $N=40$ and $M=5 \cdot 10^{4}$.

problem has a purely real spectrum, among the zeros of the approximating polynomial the ones with a small imaginary part are chosen and those which are closer to the number $\operatorname{Re} \lambda_{0}^{(n)}$ than to any other of the numbers $\operatorname{Re} \lambda_{0}^{(k)}, k \neq n$ are stored. The obtained results are presented in Table 2 together with the used values of $\lambda_{0}$ for the spectral shift and the exact eigenvalues. In the first column of the table we combine the results produced by the MATSLISE package with the exact eigenvalues because in this case all the presented digits coincide.

As can be seen from Table 2, the spectral shift technique allows us to significantly improve the obtained results for the higher eigenvalues. From now on we present only the results obtained with the help of the spectral shift technique and specify the implemented rules for choosing spectral shifts.

Example 7.2. The second example is the Boyd equation. Consider the following spectral problem [4, Example 3]

$$
\left\{\begin{array}{l}
-y^{\prime \prime}-\frac{1}{x} y=\lambda y, \quad 0<x \leq 1 \\
y(1, \lambda)=0
\end{array}\right.
$$

This equation fits into the general scheme if one takes $l=0$. We take the function $u_{0}=\sqrt{x} J_{1}(2 \sqrt{x})$ as a particular solution of (2.7) and calculate eigenvalues using the SPPS method with $N=40$ and $M=50000$. For problem (7.3) the characteristic equation is known and is given by

$$
k M_{k, 1 / 2}(2 i \sqrt{\lambda})=0
$$

where $M_{k, 1 / 2}$ is the Whittaker function and $k=k(\lambda)=(2 i \sqrt{\lambda})^{-1}$, see [4, Example 3]. In Table 3 we present the obtained results together with the exact eigenvalues calculated from (7.4) and the results obtained using SLEIGN2 and MATSLISE software. Note that some eigenvalues caused problems for the MATSLISE package, despite the excellent accuracy of the rest of the results. To simplify the reading, in the presented numbers we truncated some digits which do not coincide with the correct ones. Relative errors of the first 50 eigenvalues obtained by SPPS, MATSLISE and SLEIGN2 are presented on Figure 1, We emphasize a relative stability of the numerical results delivered by the SPPS method in comparison to those computed by MATSLISE and SLEIGN2. We 


\begin{tabular}{ccccc}
\hline$n$ & $\lambda_{n}$ (SPPS) & \multicolumn{1}{c}{$\lambda_{n}$ (exact) } & \multicolumn{1}{c}{$\lambda_{n}$ (SLEIGN2) } & $\lambda_{n}$ (MATSLISE) \\
\hline 1 & 7.3739850151752 & 7.3739850151751 & 7.37398499 & 7.3739850151751 \\
2 & 36.3360195952325 & 36.3360195952318 & 36.33601959522 & 36.3360195952318 \\
3 & 85.292582094149 & 85.292582094137 & 85.29258240 & 85.292582094137 \\
4 & 154.098623739770 & 154.098623739767 & 154.098623739742 & 154.098623739767 \\
5 & 242.705559362903 & 242.705559362911 & 242.705559362924 & 242.705559362911 \\
6 & 351.091167129407 & 351.091167129418 & 351.09116712937 & 351.091167129418 \\
8 & 627.155044324547 & 627.155044324564 & 627.155033 & 627.155044324564 \\
10 & 982.239093680177 & 982.239093680188 & 982.239069 & 982.239093680188 \\
13 & 1662.98063088581 & 1662.98063088578 & 1662.9806308832 & 1662.76 \\
20 & 3942.42966385096 & 3942.42966385102 & 3942.18 & 3942.42966385102 \\
28 & 7732.02180519217 & 7732.02180519214 & 7732.021805177 & 7729.4 \\
30 & 8876.82700072940 & 8876.82700072941 & 8876.8270009 & 8876.82700072941 \\
35 & 12084.29442705883 & 12084.29442705875 & 12084.263 & 12083.98 \\
40 & 15785.2626475018 & 15785.2626475007 & 15784.3 & 15785.2626475007 \\
50 & 24667.683593322 & 24667.683593313 & 24667.683593398 & 24662.5 \\
\hline
\end{tabular}

Table 3: The eigenvalues from Example 7.2, calculated with the use of the spectral shift technique with $N=40, M=50000$. The spectral shift is given by $\lambda_{0}^{(n)}=50 n+(2 n+0.5) i$.

mention also that the same problem was considered in [3] where less accurate results were reported: $\lambda_{1}=7.37398502, \lambda_{2}=36.3360196, \lambda_{3}=85.2925811, \lambda_{4}=154.098619$ and $\lambda_{5}=242.705545$.

The SPPS representation allows one to calculate easily the approximate eigenfunctions. The eigenfunctions that were obtained for this example are shown in Fig. 2.

Example 7.3. Consider the following spectral problem [6, Example 2].

$$
\left\{\begin{array}{l}
-y^{\prime \prime}+\left(\frac{\nu^{2}-\frac{1}{4}}{x^{2}}+x^{2}\right) y=\lambda y, \quad 0<x \leq \pi \\
y(\pi, \lambda)=0
\end{array}\right.
$$

The parameter $\nu$ was chosen equal to 2 . In this example we computed both partial and general solutions using the SPPS representations. Since the exact partial solution satisfying the asymptotic condition (2.3) is known and is given by $4 \sqrt{x} I_{1}\left(\frac{x^{2}}{2}\right)$, we compared the approximate solution with the exact one. For $N=40$ and $M=50000$ the absolute error was less than $7 \cdot 10^{-16}$. With the aid of Maple software we found that the characteristic equation of problem (7.5) has the form

$$
\frac{1}{\sqrt{\pi}} M_{\lambda / 4,1}\left(\pi^{2}\right)=0
$$

where $M_{\lambda / 4,1}$ is the Whittaker function, and used equation (7.6) to compute the exact eigenvalues. In Table 4 we present the results obtained by the SPPS method, exact eigenvalues and the results from [6], MATSLISE and SLEIGN2. As in Example 7.1 here again all presented digits in the exact eigenvalues coincide with the results delivered by MATSLISE. The performance of the SPPS method was considerably better than that of SLEIGN2 and even the 50th eigenvalue was computed correctly to 9 decimal places.

Example 7.4. Consider a particular case of the hydrogen atom equation [6, Example 4].

$$
\left\{\begin{array}{l}
-y^{\prime \prime}+\left(\frac{c}{x^{2}}+\frac{1}{x}\right) y=\lambda y, \quad 0<x \leq \pi \\
y(\pi, \lambda)=0
\end{array}\right.
$$




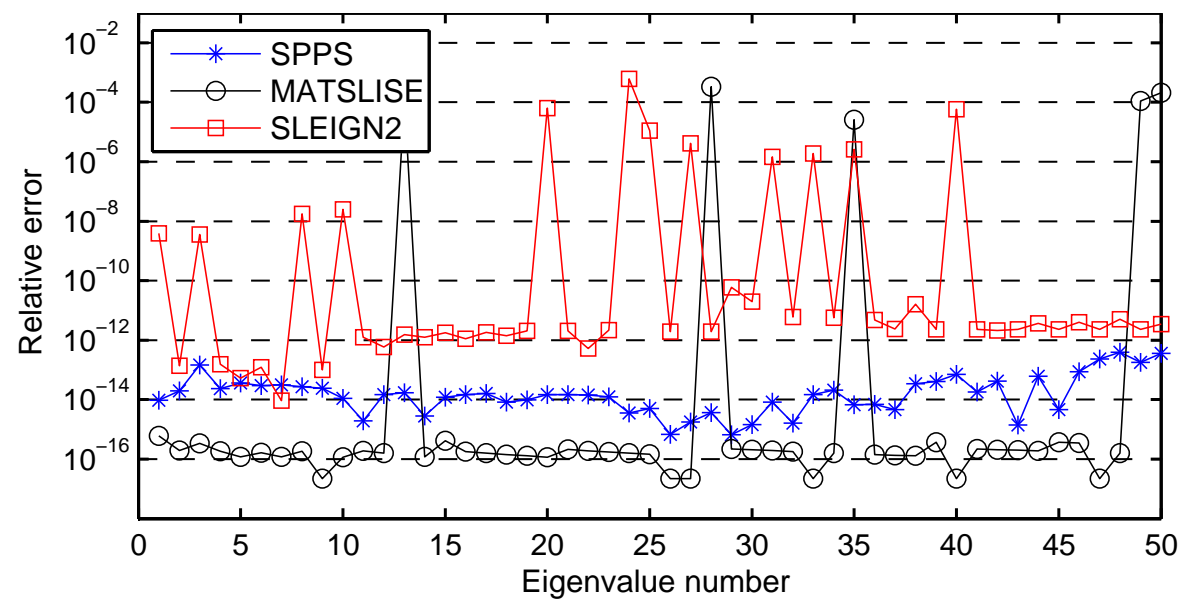

Figure 1: Relative error of the first 50 eigenvalues of the spectral problem for the Boyd equation (7.3) calculated with SPPS, SLEIGN2 and MATSLISE.

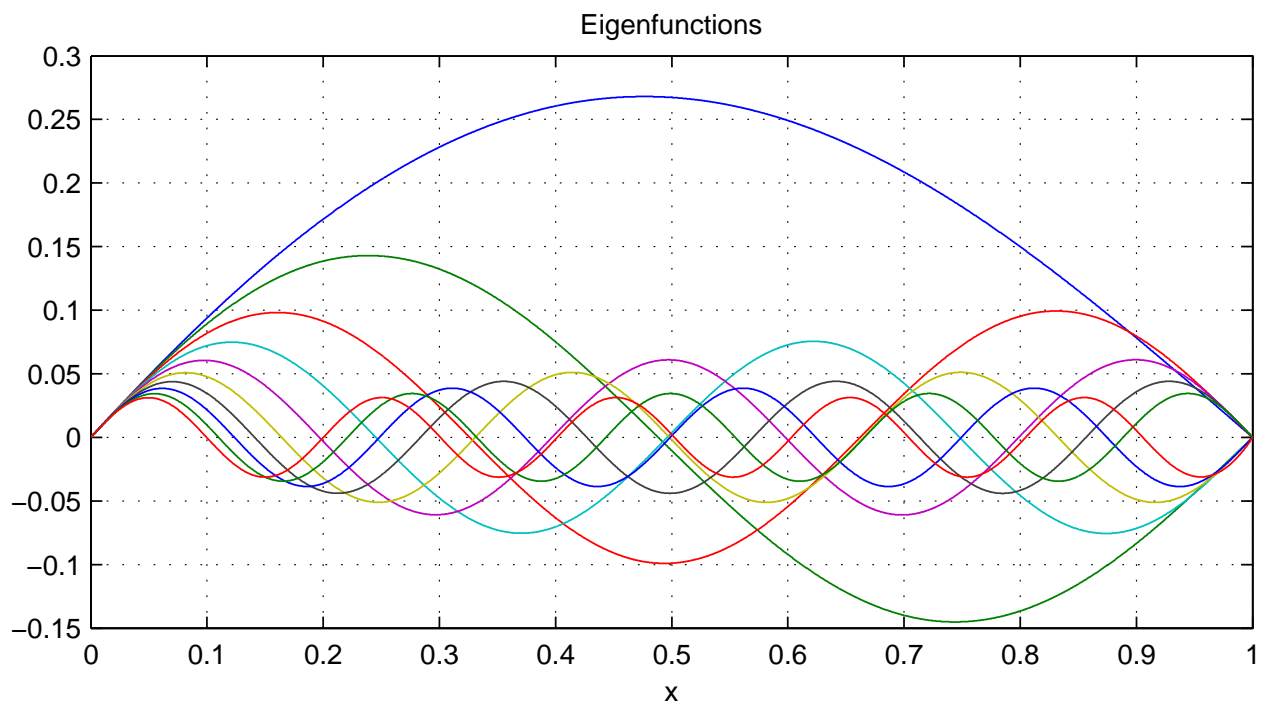

Figure 2: The first 10 eigenfunctions of problem (7.3) calculated with the use of the SPPS method.

\begin{tabular}{|c|c|c|c|c|}
\hline$n$ & $\sqrt{\lambda_{n}}(\mathrm{SPPS})$ & $\sqrt{\lambda_{n}}($ Exact/MATSLISE) & $\sqrt{\lambda_{n}}$ (SLEIGN2) & $\sqrt{\lambda_{n}}([6])$ \\
\hline 1 & 2.4629499739737 & 2.4629499739740 & 2.46295003 & 2.462949030 \\
\hline 2 & 3.2883529299493 & 3.2883529299426 & 3.28835311 & 3.288339398 \\
\hline 3 & 4.1498642187456 & 4.1498642187448 & 4.14986471 & 4.149833151 \\
\hline 4 & 5.0636688237348 & 5.0636688237341 & 5.0636695 & 5.063634795 \\
\hline 5 & 6.0075814581165 & 6.0075814581160 & 6.0075836 & 6.007577378 \\
\hline 7 & 7.9397373768999 & 7.9397373768993 & 7.939745 & \\
\hline 10 & 10.8861250916182 & 10.8861250916173 & 10.886149 & \\
\hline 15 & 15.8426318195682 & 15.8426318195682 & 15.84275 & \\
\hline 20 & 20.8202301908125 & 20.8202301908124 & 20.82057 & \\
\hline 30 & 30.7973502195887 & 30.7973502195868 & 30.7989 & \\
\hline 50 & 50.77867680977 & 50.77867680951 & 50.789 & \\
\hline
\end{tabular}

Table 4: The eigenvalues from Example 7.3, calculated with the use of the spectral shift technique with $N=50, M=50000$. The spectral shift is given by given by $\lambda_{0}^{(n)}=10 n+(n+1) i$. 


\begin{tabular}{cclll}
\hline$n$ & \multicolumn{1}{c}{$\sqrt{\lambda_{n}}$ (SPPS) } & $\sqrt{\lambda_{n}}$ (exact/MATSLISE) & $\sqrt{\lambda_{n}}$ (SLEIGN2) & \multicolumn{1}{c}{$\sqrt{\lambda_{n}}([6])$} \\
\hline 1 & 1.97027445061574 & 1.97027445061572 & 1.9702743 & 1.970274439470 \\
2 & 3.00436042551872 & 3.00436042551857 & 3.0043600 & 3.004360435708 \\
3 & 4.01515351791731 & 4.01515351791736 & 4.015153523 & 4.015153641332 \\
4 & 5.0193472218607 & 5.0193472218612 & 5.0193459 & 5.019347630098 \\
5 & 6.0210053515482 & 6.0210053515488 & 6.0210049 & 6.021006315094 \\
7 & 8.0215089715470 & 8.0215089715478 & 8.0215092 & \\
10 & 11.0202653559392 & 11.0202653559399 & 11.0202663 & \\
15 & 16.0176675547289 & 16.0176675547294 & 16.0176656 & \\
20 & 21.0155251794158 & 21.0155251794156 & 21.0155504 & \\
30 & 31.0125189152594 & 31.0125189152597 & 31.01269 & \\
50 & 51.00916429569 & 51.00916429551 & 51.0097 & \\
\hline
\end{tabular}

Table 5: The values of $\sqrt{\lambda_{n}}$ from Example 7.4, calculated with the use of the spectral shift technique with $N=40, M=50000$. The spectral shift is given by $\lambda_{0}^{(n)}=10 n+(n+1) i$.

We have chosen $c=6$ and found with the help of Maple the characteristic equation of problem (17.7)

$$
\frac{i}{8 \lambda \sqrt{\lambda}} M_{\frac{i}{2 \sqrt{\lambda}}, \frac{5}{2}}(2 i \sqrt{\lambda} \pi)=0
$$

where $M_{\frac{i}{2 \sqrt{\lambda}}, \frac{5}{2}}$ is the Whittaker function. In this example we computed both partial and general solutions using the SPPS representations. The procedure for computing the eigenvalues for this example is completely analogous to what was described above. In Table 5 we present the results obtained by the SPPS method, exact eigenvalues and the results from [6], MATSLISE and SLEIGN2. Again all presented digits in the exact eigenvalues coincide with the results delivered by MATSLISE. The SPPS method and SLEIGN2 performed similarly to the previous example.

The next example shows that the situation may change significantly when another value of the parameter $c$ is chosen.

Example 7.5. Consider the same problem as in Example 7.4 but $c=-1 / 4$ which corresponds to $l=-1 / 2$ in (2.6). The computation of eigenvalues for problem (7.7) performed by MATSLISE took several hours on Intel i7-3770 microprocessor meanwhile the computation time required by SLEIGN2 and SPPS did not change significantly. We present the relative error of the first 50 eigenvalues on Figure 3. For the SPPS method we used $N=40$ and $M=1000000$. The spectral shift was given by $\lambda_{0}^{(n)}=10 n+(n+1) i$. The accuracy of the results delivered by MATSLISE was considerably lower and once again we emphasize the stability of the accuracy of the eigenvalues computed by the SPPS method in comparison to SLEIGN2. It is worth mentioning that not only for the extreme value $l=-1 / 2$ but also for the values close to $-1 / 2$ the computation time required by the MATSLISE package increases significantly.

Example 7.6. Consider the following spectral problem [6, Example 3].

$$
\left\{\begin{array}{l}
-y^{\prime \prime}+\left(\frac{2}{x^{2}}+\sin x\right) y=\lambda y, \quad 0<x \leq \pi \\
y(\pi, \lambda)=0
\end{array}\right.
$$

For this problem we were unable to find an exact characteristic equation. We computed both particular and general solutions using the SPPS representations. The obtained results are presented in Table 6. The eigenvalues computed by the SPPS method are very close to those delivered by MATSLISE. 


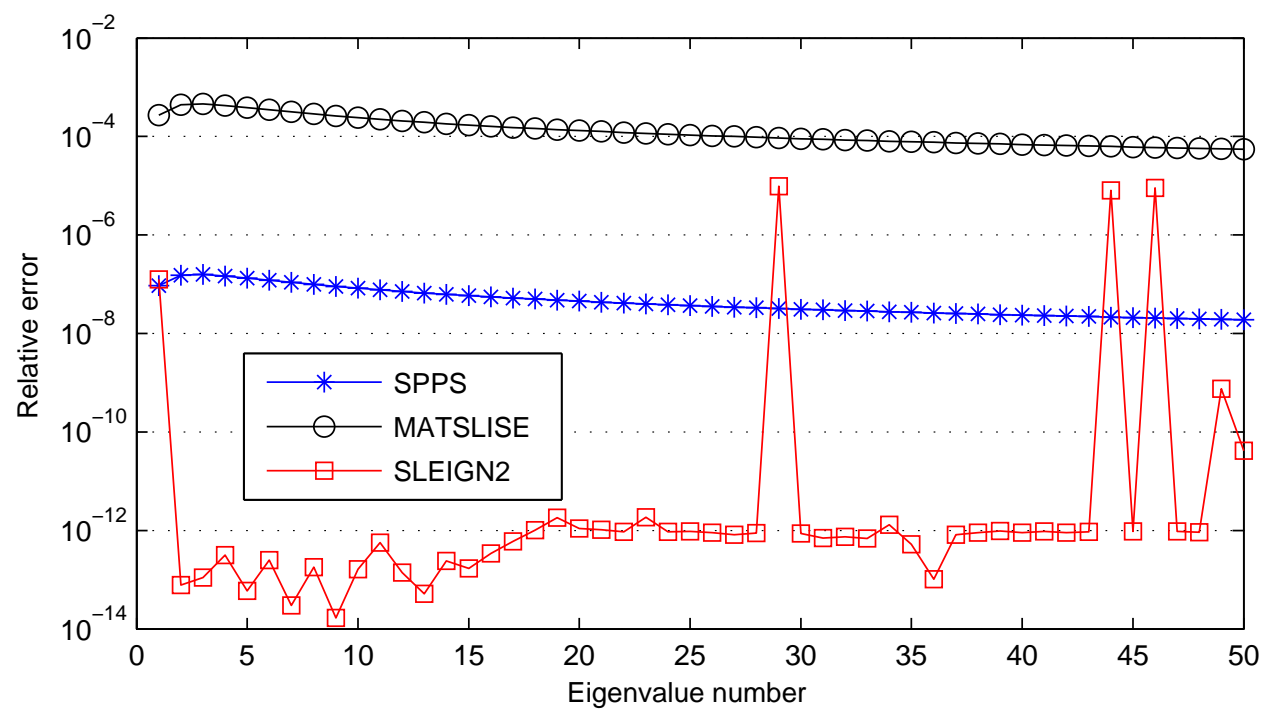

Figure 3: Relative error of the first 50 eigenvalues of problem (7.7) with $c=-1 / 4$ calculated with SPPS, SLEIGN2 and MATSLISE.

\begin{tabular}{ccccc}
\hline$n$ & $\sqrt{\lambda_{n}}$ (SPPS) & $\sqrt{\lambda_{n}}$ (MATSLISE) & $\sqrt{\lambda_{n}}$ (SLEIGN2) & Results from [6] \\
\hline 1 & 1.69965392162508 & 1.69965392162512 & 1.69965496630163 & 1.699674822427 \\
2 & 2.60438727880122 & 2.60438727880111 & 2.60439344911062 & 2.604506077325 \\
3 & 3.56972957088682 & 3.56972957088910 & 3.56974717663817 & 3.570068095387 \\
4 & 4.55232022604084 & 4.55232022604096 & 4.55235767643976 & 4.553053525686 \\
5 & 5.54189892161891 & 5.54189892161906 & 5.54196736047125 & 5.543261224280 \\
7 & 7.53001773432564 & 7.53001773432606 & 7.53019003621712 & \\
10 & 10.5211087141260 & 10.5211087141255 & 10.5215767716971 & \\
15 & 15.5141539227764 & 15.5141539227760 & 15.5156303086973 & \\
20 & 20.5106568768322 & 20.5106568768319 & 20.5139986174471 & \\
30 & 30.5071385063024 & 30.5071385063018 & 30.5174365365478 & \\
50 & 50.5043027454211 & 50.5043027452760 & 50.5434201591972 & \\
\hline
\end{tabular}

Table 6: The values of $\sqrt{\lambda_{n}}$ from Example 7.6, calculated with the use of the spectral shift technique with $N=40, M=50000$. The spectral shift is given by $\lambda_{0}^{(n)}=10 n+(n+1) i$. 


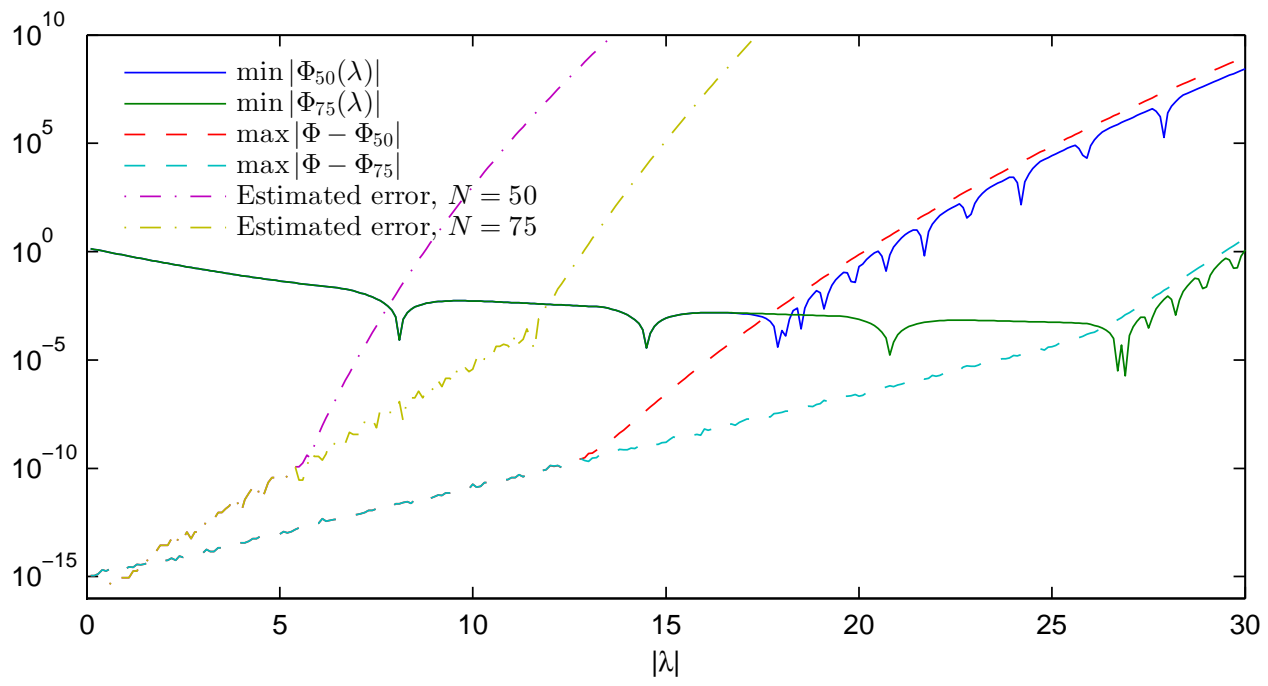

Figure 4: The graphs of $\min _{|\lambda|=r}\left|\Phi_{N}(\lambda)\right|, \max _{|\lambda|=r}\left|\Phi(\lambda)-\Phi_{N}(\lambda)\right|$ and estimate (2.16) as an illustration for Rouché's theorem in Example 7.7 for $N=50$ and $N=75$.

\subsection{Complex spectrum}

Numerical tests discussed in the previous subsection show that the SPPS method is highly competitive with the best existing codes on their field of applicability. It delivers stable and reliable results even though there remains still plenty of room for improving different computational aspects of the developed programs which implement the SPPS method. Moreover, the range of applicability of the SPPS method to the difference from the other considered codes includes complex coefficients, differential operator on the right-hand side of (2.6) and complex eigenvalues. Here we present one such example.

Example 7.7. Consider the following spectral problem with the right-hand side of the equation involving a derivative

$$
\left\{\begin{array}{l}
-y^{\prime \prime}+\frac{l(l+1)}{x^{2}} y=\lambda y^{\prime}, \quad 0<x \leq 1 \\
y^{\prime}(1, \lambda)=0 .
\end{array}\right.
$$

Using Maple we found that the solution of equation (7.10) satisfying the asymptotic condition (2.3) is given by the expression

$$
y(x ; \lambda)=\frac{2^{2 l+1} \Gamma(l+3 / 2)}{\lambda^{l+1 / 2}} \sqrt{x} e^{-\lambda x / 2} I_{l+1 / 2}\left(\frac{\lambda x}{2}\right),
$$

where $I$ is the modified Bessel function of the first kind. The derivative of this solution has the form

$$
y^{\prime}(x ; \lambda)=-\frac{2^{2 l} \Gamma(l+3 / 2) e^{-\lambda x / 2}}{\lambda^{l+1 / 2} \sqrt{x}}\left((2 l+\lambda x) I_{l+1 / 2}\left(\frac{\lambda x}{2}\right)-\lambda x I_{l-1 / 2}\left(\frac{\lambda x}{2}\right)\right) .
$$

For a numerical experiment we have chosen $l=1 / 2$, hence the exact characteristic equation is

$$
\Phi(\lambda):=-\frac{2 e^{-\lambda / 2}}{\lambda}\left((1+\lambda) I_{1}\left(\frac{\lambda}{2}\right)-\lambda I_{0}\left(\frac{\lambda}{2}\right)\right)=0 .
$$

When a problem admits complex eigenvalues we need to distinguish which roots of the polynomial $\Phi_{N}(\lambda)$ correspond to the eigenvalues and which are spurious roots appearing due to the 

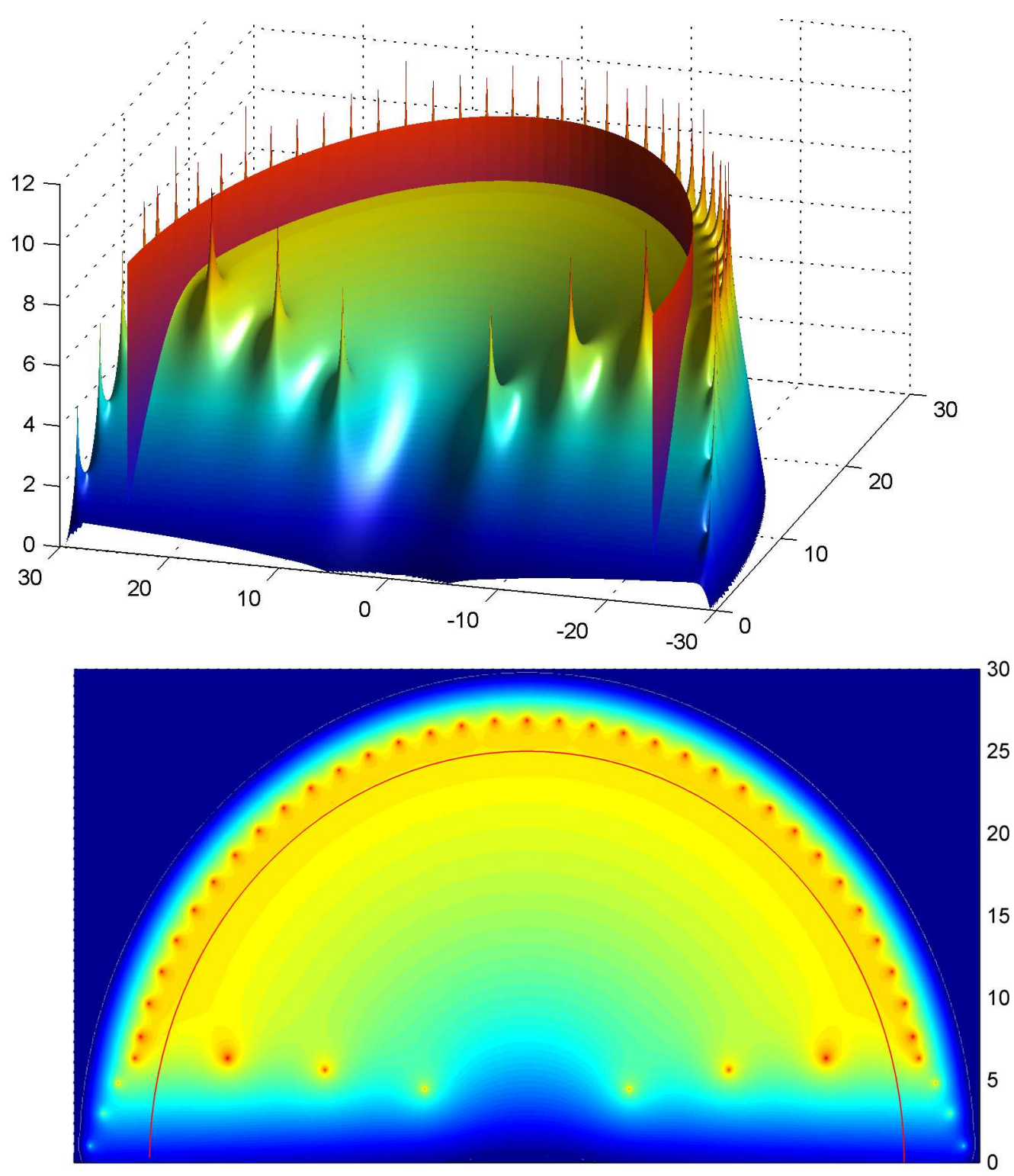

Figure 5: The image above corresponds to the graph of $-\log \left|\Phi_{75}(\lambda)\right|$. The semicylinder marks the boundary of the disk $|\lambda|=24$ illustrating the region of applicability of Rouché's theorem, see Example 7.7. The six peaks inside the region represent approximate eigenvalues. The rest of the peaks correspond to the roots of the polynomial $\Phi_{75}(\lambda)$ appearing due to the truncation procedure and do not approximate the true eigenvalues of the problem.

The image below is the top view of the one above. 


\begin{tabular}{ccc}
\hline$n$ & $\lambda_{n}$ (SPPS) & \multicolumn{1}{c}{$\lambda_{n}$ (Exact) } \\
\hline 1 & $4.47123493365+6.76481747492 i$ & $4.47123493371+6.76481747480 i$ \\
2 & $5.63553225528+13.37799928406 i$ & $5.63553225515+13.37799928396 i$ \\
3 & $6.35749327967+19.82515033089 i$ & $6.35749327947+19.82515033081 i$ \\
4 & $6.88515096019+26.20887598267 i$ & $6.88515095992+26.20887598266 i$ \\
5 & $7.30184486323+32.56088281571 i$ & $7.30184486294+32.56088281579 i$ \\
10 & $8.62739882797+64.14303168943 i$ & $8.62739882786+64.14303168978 i$ \\
20 & $9.98333956705+127.0816376255 i$ & $9.98333956726+127.0816376257 i$ \\
30 & $10.7844002548+189.9555609957 i$ & $10.7844002552+189.9555609955 i$ \\
50 & $11.7983559318+315.6569255418 i$ & $11.7983559297+315.6569255437 i$ \\
75 & $12.6055406455+472.7574366522 i$ & $12.6055406452+472.7574366509 i$ \\
100 & $13.1790674123+629.8482784849 i$ & $13.1790674160+629.8482784850 i$ \\
\hline
\end{tabular}

Table 7: The values of $\lambda_{n}$ from Example 7.7 calculated with the use of the spectral shift technique, $M=200000$ and $N=50$.

truncation procedure. Contrary to the case of a purely real spectrum we cannot simply discard all roots whose imaginary part is greater than some $\varepsilon$. Instead, Rouché's theorem and estimate (2.16) suggest that the roots closest to the origin (or to the current centre $\lambda_{0}$ when the spectral shift is used) cannot be the spurious roots. We give an illustration of application of this theorem. According to Rouché's theorem we need to find such values of the radius $r$ that

$$
\min _{|\lambda|=r}\left|\Phi_{N}(\lambda)\right|>\max _{|\lambda|=r}\left|\Phi(\lambda)-\Phi_{N}(\lambda)\right|
$$

establishing that the numbers of zeros of the functions $\Phi_{N}(\lambda)$ and $\Phi(\lambda)$ coincide inside the disk $|\lambda|<r$. We calculate $\min _{|\lambda|=r}\left|\Phi_{N}(\lambda)\right|$ using the SPPS representation. To estimate the difference $\left|\Phi(\lambda)-\Phi_{N}(\lambda)\right|$ we use (2.16). According to Lemma 2.1, for problem (7.10) one can take $C=3 / 2$. We present the obtained results on Figure 4 for $N=50$ and $N=75$ where it is compared to $\max _{|\lambda|=r}\left|\Phi(\lambda)-\Phi_{N}(\lambda)\right|$ calculated with the aid of the exact characteristic function (7.11). As can be seen from the graphs, when we use the exact characteristic function for the estimation of the error the largest values of $r$ for which inequality (17.12) holds are $r_{50} \approx 17$ and $r_{75} \approx 25$. The estimate (2.16) delivers rougher estimates, $r_{50}^{\prime} \approx 7$ and $r_{75}^{\prime} \approx 12$. On Figure 5 we show the graph of $-\log \left|\Phi_{75}(\lambda)\right|$ together with the boundary of the disk $|\lambda|=24$. The peaks on the graph correspond to the roots of the polynomial $\Phi_{75}$. Only six of them are located in the disk and hence should be considered as approximations to the eigenvalues. All other roots are located outside the disk and hence should be discarded.

To calculate the higher order eigenvalues, we applied the spectral shift technique described in Section 5. The following strategy for choosing the values of the spectral shift was implemented. Let $\lambda_{*}^{(n)}$ be the spectral shift on the $n$-th step and $\lambda_{1}, \ldots, \lambda_{n}$ the already found eigenvalues. Based on the representations (5.7) and (5.8) we calculate the roots of the polynomial $\Phi_{N}^{(n)}(\lambda)$ and reorder them with respect to the distance from $\lambda_{*}^{(n)}$. From these ordered roots we choose the closest to the point $\lambda_{*}^{(n)}$ and sufficiently distant from $\lambda_{1}, \ldots, \lambda_{n}$. We denote this root as $\lambda_{n+1}$ and set $\lambda_{*}^{(n+1)}=\lambda_{n+1}+\Delta \lambda$, where $\Delta \lambda$ is a fixed displacement. Performing this procedure with $\lambda_{*}^{(0)}=0$, $\Delta \lambda=-i, N=50$ and $M=200000$ we obtained the list of eigenvalues presented in Table 7 . The eigenvalues are ordered according to their distance to the origin. As can be seen from the table the approximate eigenvalues are calculated with a remarkable accuracy. 


\section{Acknowledgements}

We thank our colleague R. Michael Porter for providing us with a first version of a 6 points NewtonCottes Matlab numerical integration routine. R. Castillo would like to thank the support of the SIBE and EDI programs of the IPN as well as that of the project SIP 20120438. Research of V. Kravchenko and S. Torba was partially supported by CONACYT, Mexico via the project 166141.

\section{References}

[1] M. Abramovitz, I. A. Stegun. Handbook of mathematical functions, New York: Dover , 1972.

[2] N. I. Akhiezer, On the theory of coupled integral equations, Zap. Mat. Otdel. Fiz.-Mat. Fak. KhGU i KhMO 25 (1957), 5-21. (in Russian)

[3] W. Auzinger, E. Karner, O. Koch and E. Weinmüller. Collocation methods for the solution of eigenvalue problems for singular ordinary differential equations, Opuscula Mathematica, 26 (2006), No. 2, 229-241.

[4] P. B. Bailey, W. N. Everitt and A. Zettl, Computing eigenvalues of singular Sturm-Liouville problems, Results in Mathematics, 20 (1991), 391-423.

[5] P. B. Bailey, W. N. Everitt and A. Zettl, The SLEIGN2 Sturm-Liouville Code, ACM Trans. Math. Software, 21 (2001), 143-192.

[6] A. Boumenir, B. Chanane, Computing eigenvalues of Sturm-Liouville systems of Bessel type, Proceedings of the Edinburgh Mathematical Society, 42 (1999), 257-265.

[7] H. M. Campos, V. V. Kravchenko and L. M. Mendez, Complete families of solutions for the Dirac equation: an application of bicomplex pseudoanalytic function theory and transmutation operators, Advances in Applied Clifford Algebras 22 (2012), no. 3, 577-594.

[8] H. Campos, V. V. Kravchenko and S. M. Torba, Transmutations, L-bases and complete families of solutions of the stationary Schrödinger equation in the plane, J. Math. Anal. Appl. 389 (2012), no. 2, 1222-1238.

[9] R. Castillo, K. V. Khmelnytskaya, V. V. Kravchenko and H. Oviedo, Efficient calculation of the reflectance and transmittance of finite inhomogeneous layers, J. Opt. A: Pure and Applied Optics 11 (2009), 065707.

[10] R. Castillo P., V. V. Kravchenko, H. Oviedo and V. S. Rabinovich, Dispersion equation and eigenvalues for quantum wells using spectral parameter power series, J. Math. Phys., 52 (2011), 043522 (10 pp.)

[11] H. Chébli, A. Fitouhi and M. M. Hamza, Expansion in series of Bessel functions and transmutations for perturbed Bessel operators, J. Math. Anal. Appl. 181 (1994), no. 3, 789-802.

[12] J. B. Conway, Functions of one complex variable. Second edition. Graduate Texts in Mathematics, 11. New York-Berlin: Springer-Verlag, 1978.

[13] P. J. Davis, P. Rabinowitz, Methods of numerical integration. Second edition, New York: Dover Publications, 2007.

[14] J. Delsarte, J. L. Lions, Transmutations d'opérateurs différentiels dans le domaine complexe, Comment. Math. Helv. 32 (1956), 113-128.

[15] L. Erbe, R. Mert and A. Peterson, Spectral parameter power series for Sturm-Liouville equations on time scales, Applied Mathematics and Computation, 218 (2012) 7671-7678.

[16] J.-C. Guillot, J. V. Ralston, Inverse spectral theory for a singular Sturm-Liouville operator on [0,1], J. Differential Equations 76 (1988), no. 2, 353-373.

[17] Ph. Hartman, Ordinary Differential Equations, New York-London-Sydney: John Wiley \& Sons, 1964.

[18] K. V. Khmelnytskaya, V. V. Kravchenko and J. A. Baldenebro-Obeso, Spectral parameter power series for fourth-order Sturm-Liouville problems, Applied Mathematics and Computation, 219 (2012), 3610-3624.

[19] K. V. Khmelnytskaya, V. V. Kravchenko and H. C. Rosu, Eigenvalue problems, spectral parameter power series, and modern applications. Submitted, available at arXiv:1112.1633.

[20] K. V. Khmelnytskaya, V. V. Kravchenko, S. M. Torba and S. Tremblay, Wave polynomials and Cauchy's problem for the Klein-Gordon equation, J. Math. Anal. Appl., 399 (2013), 191-212.

[21] K. V. Khmelnytskaya, H. C. Rosu, A new series representation for Hill's discriminant, Annals of Physics 325 (2010) 2512-2521. 
[22] K. V. Khmelnytskaya, I. Serroukh, The heat transfer problem for inhomogeneous materials in photoacoustic applications and spectral parameter power series. Mathematical Methods in the Applied Sciences (2013), DOI: 10.1002/mma.2732.

[23] K. V. Khmelnytskaya, T. V. Torchynska, Reconstruction of potentials in quantum dots and other small symmetric structures, Mathematical Methods in the Applied Sciences 33 (2010), Issue 4, 469-472.

[24] A. Kostenko, G. Teschl, On the singular Weyl-Titchmarsh function of perturbed spherical Schrödinger operators, J. Differential Equations 250 (2011) 3701-3739.

[25] V. V. Kravchenko, A representation for solutions of the Sturm-Liouville equation, Complex Variables and Elliptic Equations 53 (2008) 775-789.

[26] V. V. Kravchenko, Applied pseudoanalytic function theory. Basel: Birkhäuser, Series: Frontiers in Mathematics, 2009.

[27] V. V. Kravchenko, R. M. Porter, Spectral parameter power series for Sturm-Liouville problems, Mathematical Methods in the Applied Sciences 33 (2010), 459-468.

[28] V. V. Kravchenko, S. M. Torba, Spectral problems in inhomogeneous media, spectral parameter power series and transmutation operators, in 2012 International Conference on Mathematical Methods in Electromagnetic Theory (MMET), IEEE Conference Publications, pp.18-22, doi:10.1109/MMET.2012.6331232.

[29] V. V. Kravchenko, S. M. Torba, Transmutations and spectral parameter power series in eigenvalue problems, Operator Theory: Advances and Applications, 228 (2013), 209-238.

[30] V. V. Kravchenko, S. M. Torba, Construction of transmutation operators and hyperbolic pseudoanalytic functions. Submitted, available from arXiv:1208.6166, $37 \mathrm{pp.}$

[31] V. V. Kravchenko, S. M. Torba, Numerical solution of spectral problems using transmutations, Submitted.

[32] V. V. Kravchenko, U. Velasco-García, Dispersion equation and eigenvalues for the Zakharov-Shabat system using spectral parameter power series. Journal of Mathematical Physics, 52 (2011), issue 6, \#063517, 8 pp.

[33] V. Ledoux, M. Van Daele and G. Vanden Berghe, Matslise: A Matlab package for the Numerical Solution of Sturm-Liouville and Schrödinger equations, ACM Transactions on Mathematical Software, 31 (2005), 532-554.

[34] B. M. Levitan, Inverse Sturm-Liouville problems, VSP, Zeist, 1987.

[35] G. Pólya, On the mean value theorem corresponding to a given linear homogeneous differential equation, Trans. Amer. Math. Soc. 24 (1924), 312-324.

[36] V. S. Rabinovich, R. Castillo-Pérez and F. Urbano-Altamirano, On the essential spectrum of quantum waveguides, Mathematical Methods in the Applied Sciences (2013), DOI: 10.1002/mma.2623.

[37] M. Rajović, R. Stojiljković, The first, the second, and the third Liouville formula and periodical solutions of linear differential equation of the second order. Kragujevac J. Math. 30 (2007), 131-139.

[38] S. M. Sitnik, Transmutations and applications: a survey, arXiv:1012.3741v1, originally published in the book: Advances in Modern Analysis and Mathematical Modeling (Editors: Yu.F.Korobeinik, A.G.Kusraev, Vladikavkaz: Vladikavkaz Scientific Center of the Russian Academy of Sciences and Republic of North OssetiaAlania, 2008, 226-293).

[39] V. V. Stashevskaya, On the inverse problem of spectral analysis for a differential operator with a singularity at zero, Zap. Mat. Otdel. Fiz.-Mat. Fak. KhGU i KhMO 25 (1957), no. 4, 49-86. (in Russian)

[40] V. Ya. Volk, On inversion formulas for a differential equation with a singularity at $x=0$, Uspehi Matem. Nauk (N.S.), 8 (1953). no. 4(56), 141-151.

[41] J. Weidmann, Spectral Theory of Ordinary Differential Operators, Lecture Notes in Math., Berlin: Springer, vol. $1258,1987$.

[42] A. Zettl, Sturm-Liouville theory, Mathematical Surveys and Monographs, 121. Providence, RI: American Mathematical Society, 2005. 
This figure "CharEq150a.png" is available in "png" format from: http://arxiv.org/ps/1303.3911v1 
This figure "CharEq150top.png" is available in "png" format from: http://arxiv.org/ps/1303.3911v1 